\title{
Molecular line study of the very young protostar IRAM 04191 in Taurus: infall, rotation, and outflow
}

\author{
A. Belloche ${ }^{1}$, P. André ${ }^{1}$, D. Despois ${ }^{2}$, and S. Blinder ${ }^{2,3}$ \\ 1 Service d'Astrophysique, CEA/DSM/DAPNIA, C.E. Saclay, 91191, Gif-sur-Yvette Cedex, France \\ 2 Observatoire de Bordeaux (INSU/CNRS), BP 89, 33270 Floirac, France \\ ${ }^{3}$ Division of Nuclear Medicine, Vancouver Hospital and Health Sciences Center, Vancouver, B.C., Canada
}

Received 16 April 2002 / Accepted 12 July 2002

\begin{abstract}
We present a detailed millimeter spectroscopic study of the circumstellar environment of the low-luminosity Class 0 protostar IRAM 04191+1522 in the Taurus molecular cloud. Molecular line observations with the IRAM $30 \mathrm{~m}$ telescope demonstrate that the $\sim 14000 \mathrm{AU}$ radius protostellar envelope is undergoing both extended infall and fast, differential rotation. Radiative transfer modeling of multitransition CS and $\mathrm{C}^{34} \mathrm{~S}$ maps indicate an infall velocity $v_{\text {inf }} \sim 0.15 \mathrm{~km} \mathrm{~s}^{-1}$ at $r \sim 1500$ AU and $v_{\text {inf }} \sim 0.1 \mathrm{~km} \mathrm{~s}^{-1}$ up to $r \sim 11000 \mathrm{AU}$, as well as a rotational angular velocity $\Omega \sim 3.9 \times 10^{-13} \mathrm{rad} \mathrm{s}^{-1}$, strongly decreasing with radius beyond $3500 \mathrm{AU}$ down to a value $\Omega \sim 1.5-3 \times 10^{-14} \mathrm{rad} \mathrm{s}^{-1}$ at $\sim 11000 \mathrm{AU}$. Two distinct regions, which differ in both their infall and their rotation properties, therefore seem to stand out: the inner part of the envelope $(r \lesssim 2000-4000 \mathrm{AU})$ is rapidly collapsing and rotating, while the outer part undergoes only moderate infall/contraction and slower rotation. These contrasted features suggest that angular momentum is conserved in the collapsing inner region but efficiently dissipated due to magnetic braking in the slowly contracting outer region. We propose that the inner envelope is in the process of decoupling from the ambient cloud and corresponds to the effective mass reservoir $\left(\sim 0.5 M_{\odot}\right)$ from which the central star is being built. Comparison with the rotational properties of other objects in Taurus suggests that IRAM 04191 is at a pivotal stage between a prestellar regime of constant angular velocity enforced by magnetic braking and a dynamical, protostellar regime of nearly conserved angular momentum. The rotation velocity profile we derive for the inner IRAM 04191 envelope should thus set some constraints on the distribution of angular momentum on the scale of the outer Solar system at the onset of protostar/disk formation.
\end{abstract}

Key words. stars: formation - stars: circumstellar matter - stars: rotation - stars: planetary systems: protoplanetary disks ISM: kinematics and dynamics - ISM: molecules

\section{Introduction}

\subsection{The enigmatic onset of protostellar collapse}

Despite recent progress, the initial conditions of star formation and the first phases of protostellar collapse remain poorly known (e.g. Myers 1999; André et al. 2000 for reviews). In the standard theory of isolated, low-mass star formation (e.g. Shu et al. 1987), the initial conditions correspond to essentially static singular isothermal spheroids (SISs, which have $\left.\rho=\left(a_{\mathrm{s}}{ }^{2} / 2 \pi G\right) r^{-2}\right)$, assumed to be in slow, solid-body rotation (Terebey et al. 1984 - TSC84) and weakly magnetized (e.g. Galli \& Shu 1993; Li \& Shu 1997). This leads to a strictly constant mass accretion rate, $\dot{M}_{\text {acc }} \sim a_{\mathrm{s}}{ }^{3} / G$ (where $a_{\mathrm{s}}$ is the isothermal sound speed), and to a growth of the centrifugal disk as $R_{\text {disk }} \propto t^{3}$ (cf. TSC84) during the protostellar accretion phase $(t>0)$. Other theoretical models exist, however, that predict

Send offprint requests to: A. Belloche,

e-mail: belloche@lra.ens.fr or pandre@cea.fr a time-dependent accretion history if the collapse initial conditions are either not singular or not scale-free (e.g. Foster \& Chevalier 1993; Henriksen et al. 1997; Basu 1997; Ciolek \& Königl 1998; Hennebelle et al. 2002). Starting from realistic, finite-sized prestellar cores with $\rho \approx$ const. in their central region (cf. Ward-Thompson et al. 1999; Bacmann et al. 2000; Alves et al. 2001), these models yield supersonic inward velocities close to the center prior to point mass formation (i.e. at $t<0$ ) and result in denser, nonequilibrium density distributions with strong differential rotation at the onset of the main accretion phase, i.e., at $t=0$. In these models, the accretion rate $\dot{M}_{\text {acc }}$ is initially significantly larger than in the Shu model, then quickly converges toward the standard $\sim a_{\mathrm{s}}{ }^{3} / G$ value, and finally declines much below $a_{\mathrm{s}}{ }^{3} / G$ because of the finite reservoir of mass (see, e.g., Foster \& Chevalier 1993; Henriksen et al. 1997). Conservation of angular momentum during dynamical collapse at $t<0$ produces a differential rotation profile at $t=0$ (e.g. $\Omega \propto r^{-1}$ in the magnetically-controlled model of Basu 1998). This rotation profile in turn implies a more rapid 
growth of $R_{\text {disk }}$ initially (i.e., $R_{\text {disk }} \propto t$ at small $t>0$ in the Basu model) than in the TSC84 model.

Getting at a better, more quantitative knowledge of protostellar collapse is crucial, e.g., to gain insight into the origin of stellar masses and disk formation. Observationally, there are two complementary approaches to estimating the initial conditions of protostar formation. The first approach consists in studying the structure and kinematics of "prestellar cores" such as L1544 (e.g. Ward-Thompson et al. 1999; Tafalla et al. 1998), representative of times $t \lesssim 0$. The second approach, adopted here, is the detailed study of Class 0 accreting protostars observed at $t \gtrsim 0$, such as IRAM 04191 (see Sect. 1.2), which should still retain detailed memory of their initial conditions.

\subsection{IRAM 04191: A very young Class 0 protostar}

The massive $\left(M_{\mathrm{tot}} \sim 1.5 M_{\odot}\right)$ dense core/envelope of the Class 0 object, IRAM 04191+1522 (hereafter IRAM 04191), was originally discovered in the millimeter dust continuum with the IRAM $30 \mathrm{~m}$ telescope in the southern part of the Taurus molecular cloud (André et al. 1999 - hereafter AMB99). Follow-up observations revealed a highly collimated $\mathrm{CO}$ bipolar outflow (see Fig. 1), a weak $3.6 \mathrm{~cm}$ VLA radio continuum source located at its center of symmetry, and spectroscopic evidence of spatially extended infall motions in the bulk of the envelope. These are typical attributes of a Class 0 protostar (André et al. 1993, 2000).

The very high envelope mass to luminosity ratio of IRAM $04191\left(M_{\mathrm{env}}^{<4200 \mathrm{AU}} / L_{\mathrm{bol}} \gtrsim 3 M_{\odot} / L_{\odot}\right)$ and its position in the $L_{\text {bol }}-T_{\text {bol }}$ evolutionary diagram $\left(L_{\text {bol }} \sim 0.15 L_{\odot}\right.$ and $T_{\text {bol }} \sim 18 \mathrm{~K}$ ) suggest an age $t \sim 1-3 \times 10^{4} \mathrm{yr}$ since the beginning of the accretion phase (see AMB99). This is significantly younger than all of the IRAS candidate protostars of Taurus (e.g. Kenyon et al. 1993), including L1527 which has $t \lesssim 10^{5} \mathrm{yr}$ (e.g. Ohashi et al. 1997a). IRAM 04191 thus appears to be the youngest accreting protostar known so far in Taurus, although the collapsing protostellar condensation MC 27 discovered by Onishi et al. (1999) may be in a comparable evolutionary state.

As IRAM 04191 is particularly young, nearby $(d=$ $140 \mathrm{pc}$ ), and relatively isolated, the study of its velocity structure based on molecular line observations provides a unique opportunity to set constraints on collapse models. This is especially true since the viewing angle is favorable. The $\mathrm{CO}(2-$ 1) outflow map of AMB99 (see Fig. 1) shows well separated outflow lobes with almost no overlap between blue-shifted and red-shifted emission, indicating that the flow lies out of the plane of the sky at an intermediate inclination angle (e.g. Cabrit $\&$ Bertout 1990). In addition, AMB99 estimate an aspect ratio of $\sim 0.65$ for the circumstellar dust/ $\mathrm{N}_{2} \mathrm{H}^{+}$envelope, whose major axis is perpendicular to the outflow axis (cf. Fig. 1). Both characteristics are consistent with an inclination angle of the outflow axis to the line of sight of $i \sim 50^{\circ}$.

Here, we present and discuss the results of a comprehensive set of molecular line observations toward IRAM 04191. The layout of the paper is as follows. Section 2 summarizes observational details. Section 3 interprets the observations in terms of infall, rotation, and outflow motions in the envelope.

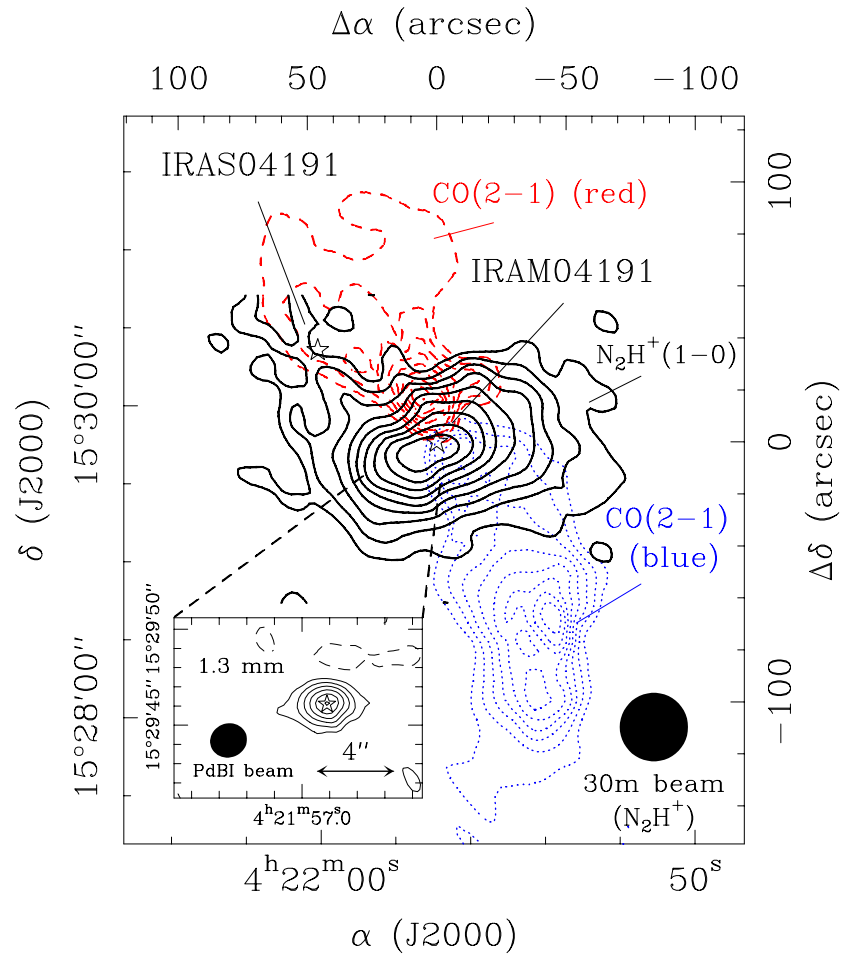

Fig. 1. $\mathrm{N}_{2} \mathrm{H}^{+}(1-0)$ integrated intensity map of the IRAM 04191 protostellar envelope overlaid on the $\mathrm{CO}(2-1)$ outflow map of AMB99. Both maps were taken with the IRAM 30 m telescope. The $\mathrm{N}_{2} \mathrm{H}^{+}(1-$ 0 ) emission is integrated over the whole seven-component multiplet from 5.7 to $22.6 \mathrm{~km} \mathrm{~s}^{-1}$ and the contours go from 0.5 to $4.5 \mathrm{~K} \mathrm{~km} \mathrm{~s}^{-1}$ by $0.5 \mathrm{~K} \mathrm{~km} \mathrm{~s}^{-1}$. The blueshifted $\mathrm{CO}(2-1)$ emission (dotted contours) is integrated over the $0-5 \mathrm{~km} \mathrm{~s}^{-1}$ velocity range, and the redshifted $\mathrm{CO}(2-1)$ emission (dashed contours) is integrated over the $8-13 \mathrm{~km} \mathrm{~s}^{-1}$ velocity range; the $\mathrm{CO}(2-1)$ contours go from 5 to $21 \mathrm{~K} \mathrm{~km} \mathrm{~s}^{-1}$ by $2 \mathrm{~K} \mathrm{~km} \mathrm{~s}^{-1}$. The insert in the bottom-left corner shows a $227 \mathrm{GHz}$ dust continuum map of the inner envelope obtained at the IRAM Plateau de Bure interferometer (see Sect. 3.1), with positive (solid) contours from +1 to +6 by $1 \mathrm{mJy} / 1.9^{\prime \prime}$-beam; the dotted contour is negative at $-1 \mathrm{mJy} / 1.9^{\prime \prime}$-beam). In both maps, the central star symbol at $(0,0)$ marks the position of IRAM 04191 as determined by a 2D Gaussian fit to the PdBI $227 \mathrm{GHz}$ continuum image. The other star symbol indicates the position of the Class I source IRAS 04191.

We then model the observed spectra using radiative transfer simulations computed in 1D spherical geometry with radial infall motions (Sect. 4) and in 2D axial geometry with both infall and rotational motions (Sect. 5). Section 6 compares the derived constraints on the velocity structure of the IRAM 04191 envelope with the predictions of collapse models. Our conclusions are summarized in Sect. 7.

\section{Observations}

We carried out millimeter line observations with the IRAM $30 \mathrm{~m}$ telescope at Pico Veleta, Spain, during 7 nights in July and August 1999, in the following molecular transitions: $\mathrm{N}_{2} \mathrm{H}^{+}(1-0), \mathrm{CS}(2-1), \mathrm{C}^{34} \mathrm{~S}(2-1), \mathrm{HCO}^{+}(1-0), \mathrm{H}^{13} \mathrm{CO}^{+}(1-0)$, $\mathrm{C}_{3} \mathrm{H}_{2}\left(2_{12}-1_{01}\right)$ at $3 \mathrm{~mm}, \mathrm{CS}(3-2), \mathrm{C}^{34} \mathrm{~S}(3-2), \mathrm{H}_{2} \mathrm{CO}\left(2_{12}-\right.$ $\left.1_{11}\right)$ at $2 \mathrm{~mm}$, and $\mathrm{CS}(5-4), \mathrm{H}_{2} \mathrm{CO}\left(3_{12}-2_{11}\right), \mathrm{C}^{18} \mathrm{O}(2-1)$, 
$\mathrm{DCO}^{+}(3-2)$ at $1.2 \mathrm{~mm}$. Our adopted set of line frequencies is given in Table 1 . The half-power beamwidths were $\sim 26^{\prime \prime}$, $\sim 17^{\prime \prime}$ and $\sim 10^{\prime \prime}$ at $3 \mathrm{~mm}, 2 \mathrm{~mm}$, and $1.2 \mathrm{~mm}$, respectively. We used four SIS heterodyne receivers simultaneously and an autocorrelation spectrometer as backend, with a spectral resolution of $20 \mathrm{kHz}$ at $3 \mathrm{~mm}$ and $40 \mathrm{kHz}$ at $2 \mathrm{~mm}$ and $1.2 \mathrm{~mm}$. The corresponding velocity resolutions ranged from 0.05 to $0.08 \mathrm{~km} \mathrm{~s}^{-1}$ per channel. The observations were done in single sideband mode, with sideband rejections of $0.01,0.1$ and 0.05 at $3 \mathrm{~mm}, 2 \mathrm{~mm}$, and $1.2 \mathrm{~mm}$, respectively. Accordingly, the calibration uncertainty was $\sim 10 \%$. The forward and beam efficiencies of the telescope used to convert antenna temperatures $T_{\mathrm{A}}^{*}$ into main beam temperatures $T_{\mathrm{mb}}$ are listed in Table 1 . The system temperatures ( $T_{\mathrm{A}}^{*}$ scale) ranged from $\sim 110 \mathrm{~K}$ to $\sim 150 \mathrm{~K}$ at $3 \mathrm{~mm}, \sim 280 \mathrm{~K}$ to $\sim 410 \mathrm{~K}$ at $2 \mathrm{~mm}$, and $\sim 300 \mathrm{~K}$ to $\sim 550 \mathrm{~K}$ at $1.2 \mathrm{~mm}$. The telescope pointing was checked every $\sim 2$ hours on Saturn, 0528+134, and/or 0420-014, and found to be accurate to $\sim 3^{\prime \prime}$ (rms). The telescope focus was optimized on Saturn every $\sim 3$ hours. Position switching observations were done with a reference position located at either $(\Delta \alpha, \Delta \delta)=\left(1200^{\prime \prime}\right.$, $\left.-1200^{\prime \prime}\right)$ or $\left(80^{\prime \prime},-80^{\prime \prime}\right)$ relative to the $(0,0)$ position (envelope center as measured in the $1.3 \mathrm{~mm}$ continuum). Extensive mapping was performed in the "on-the-fly" mode (Ungerechts et al. 2000). A few additional $\mathrm{C}^{18} \mathrm{O}(1-0), \mathrm{C}^{18} \mathrm{O}(2-1), \mathrm{CS}(3-$ 2), $\mathrm{C}^{34} \mathrm{~S}(3-2), \mathrm{CS}(5-4)$ and $\mathrm{H}^{13} \mathrm{CO}^{+}(3-2)$ position-switch observations, performed in September 1997, November 2000 and October 2001, will also be used here. All of these single-dish data were reduced with the CLASS software package (Buisson et al. 2002).

In addition, we also observed IRAM 04191 with the 5antenna IRAM Plateau de Bure interferometer (PdBI) in its B1, C2, D configurations between January and April 1999. The two receivers of each antenna were tuned to the $\operatorname{CS}(2-1)$ and $\mathrm{H}_{2} \mathrm{CO}\left(3_{12}-2_{11}\right)$ lines, with spectral resolutions of $40 \mathrm{kHz}$ and $80 \mathrm{kHz}$, corresponding to velocity resolutions of $0.12 \mathrm{~km} \mathrm{~s}^{-1}$ and $0.10 \mathrm{~km} \mathrm{~s}^{-1}$, respectively. The four remaining windows of the PdBI correlator were used to record the continuum emission with a total bandwidth of $300 \mathrm{MHz}$ at both $98 \mathrm{GHz}$ $(\lambda \sim 3 \mathrm{~mm})$ and $227 \mathrm{GHz}(\lambda \sim 1.3 \mathrm{~mm})$. The (naturallyweighted) synthesized half-power beamwidths were $4.5^{\prime \prime} \times$ 4.4" (630 AU×620 AU) at $98 \mathrm{GHz}$ and $1.9^{\prime \prime} \times 1.8^{\prime \prime}(270 \mathrm{AU} \times$ $250 \mathrm{AU}$ ) at $227 \mathrm{GHz}$, and the (FWHP) primary beams $\sim 50^{\prime \prime}$ and $\sim 25^{\prime \prime}$, respectively. The correlator bandpass was calibrated on the strong source $3 \mathrm{C} 273$. Several nearby phase calibrators were observed to determine the time-dependent complex antenna gains. The absolute calibration uncertainty was estimated to be $\sim 15 \%$. The data were calibrated and imaged using the CLIC (Lucas 1999) and Mapping (Guilloteau et al. 2002) packages in the IRAM software.

\section{Results and qualitative interpretation: Evidence for rotation and infall}

\subsection{Weak $1.3 \mathrm{~mm}$ continuum detection at $\mathrm{PdBI}$}

The PdBI continuum image at $227 \mathrm{GHz}$ (see Fig. 1) reveals weak, point-like emission centered at $\alpha_{(2000)}=04^{\mathrm{h}} 21^{\mathrm{m}} 56^{\mathrm{s}} 91$, $\delta_{(2000)}=15^{\circ} 29^{\prime} 46.1^{\prime \prime}$, a position which should be accurate to
Table 1. Adopted line rest frequencies and telescope efficiencies.

\begin{tabular}{lccccc}
\hline \hline \multicolumn{1}{c}{ Line } & $\begin{array}{c}\text { Frequency } \\
(\mathrm{MHz})\end{array}$ & $\begin{array}{c}\sigma_{\mathrm{v}}{ }^{2} \\
\left(\mathrm{~km} \mathrm{~s}^{-1}\right)\end{array}$ & $\begin{array}{c}\text { Ref. } \\
(3)\end{array}$ & $\begin{array}{c}F_{\text {eff }}{ }^{4} \\
(\%)\end{array}$ & $\begin{array}{c}B_{\text {eff }}{ }^{4} \\
(\%)\end{array}$ \\
\hline $\mathrm{C}_{3} \mathrm{H}_{2}\left(2_{12}-1_{01}\right)$ & $85338.905(6)$ & 0.02 & $(1)$ & 92 & 73 \\
$\mathrm{~N}_{2} \mathrm{H}^{+}(101-012)$ & $93176.258(7)$ & 0.02 & $(2)$ & 92 & 73 \\
$\mathrm{C}^{34} \mathrm{~S}(2-1)$ & $96412.952(1)$ & 0.003 & $(3)$ & 92 & 73 \\
$\mathrm{CS}(2-1)$ & $97980.953(1)$ & 0.003 & $(3)$ & 90 & 73 \\
$\mathrm{C}^{18} \mathrm{O}(1-0)$ & $109782.175(2)$ & 0.005 & $(4)$ & 92 & 73 \\
$\mathrm{C}^{34} \mathrm{~S}(3-2)$ & $144617.101(1)$ & 0.002 & $(3)$ & 87 & 65 \\
& & & & 90 & 54 \\
$\mathrm{CS}(3-2)$ & $146969.026(1)$ & 0.002 & $(3)$ & 90 & 54 \\
$\mathrm{C}^{18} \mathrm{O}(2-1)$ & $219560.3541(15)$ & 0.002 & $(5)$ & 86 & 42 \\
$\mathrm{CS}(5-4)$ & $244935.555(1)$ & 0.001 & $(3)$ & 84 & 42 \\
\hline
\end{tabular}

Notes: ${ }^{1}$ The frequency uncertainty in units of the last significant digit is given in parentheses.

${ }^{2}$ Frequency uncertainty converted in units of velocity.

${ }^{3}$ References for rest frequencies: (1) Laboratory measurement from Vrtilek et al. (1987); (2) Observational result from Lee et al. (2001); (3) Laboratory measurement from Gottlieb et al. (2002). (4) Laboratory measurement from Klapper (2001, private communication). (5) Laboratory measurement from Klapper et al. (2001).

${ }^{4}$ Forward and beam efficiencies of the IRAM $30 \mathrm{~m}$ telescope (Wild 1999 and http://www.iram.es/).

better than $0.5^{\prime \prime}$. (The PdBI position is offset by $\sim 5^{\prime \prime}$ from the $\mathrm{N}_{2} \mathrm{H}^{+}(1-0)$ centroid observed at the $30 \mathrm{~m}$ telescope, which is only marginally significant given the $\sim 3^{\prime \prime}$ single-dish pointing accuracy.) We measure a peak $227 \mathrm{GHz}$ flux density of $S_{\text {peak }}^{1.9^{\prime \prime}}=6.1 \pm 0.4 \mathrm{mJy} / 1.9^{\prime \prime}$-beam at the central position. The $227 \mathrm{GHz}$ emission is slightly resolved and a Gaussian fit performed in the $u v$-plane yields a deconvolved $F W H M$ size of $\left(1.1^{\prime \prime} \pm 0.4^{\prime \prime}\right) \times\left(0.6^{\prime \prime} \pm 0.3^{\prime \prime}\right)$ with a position angle $\mathrm{PA}=84^{\circ}$. At $98 \mathrm{GHz}$, the rms noise level is $0.14 \mathrm{mJy} / 4.5^{\prime \prime}$-beam and we do not detect any emission above $0.6 \mathrm{mJy} / 4.5^{\prime \prime}$-beam $(\sim 4 \sigma)^{1}$.

For comparison, using the IRAM $30 \mathrm{~m}$ telescope equipped with the MPIfR bolometer array (MAMBO), Motte \& André (2001) measured a peak flux density of $S_{\text {peak }}^{11^{\prime \prime}}=110 \pm$ $7 \mathrm{mJy} / 11^{\prime \prime}$-beam at $1.3 \mathrm{~mm}\left(v_{\text {eff }} \sim 240 \mathrm{GHz}-\right.$ e.g. Broguière et al. 2002) and a radial intensity profile of the form $I(\theta) \propto$ $\theta^{-0.6 \pm 0.1}$ in the range of angular radii $\theta=11^{\prime \prime}$ to $\theta=100^{\prime \prime}$. This extended $1.3 \mathrm{~mm}$ continuum source is clearly the dust counterpart of the circumstellar gas envelope/core observed in $\mathrm{N}_{2} \mathrm{H}^{+}$(cf. Fig. 1). Assuming the same radial intensity profile holds at smaller angular radii, one expects the peak flux density of the envelope to scale as $S_{\text {peak }}\left(\theta_{b}\right) \propto \theta_{b}^{1.4 \pm 0.1}$ with beamsize $\theta_{b}$. If we adopt a dust opacity index $\beta=1.5$ to account for the slight difference in observing frequency between the $30 \mathrm{~m}$ and PdBI measurements, this flux-density scaling pre$\operatorname{dicts} S_{\text {peak }}^{1.9^{\prime \prime}} \approx 7.7 \mathrm{mJy} / 1.9^{\prime \prime}$-beam, which is only $25 \%$ larger than the PdBI peak flux density quoted above. Given the relative calibration uncertainties, this comparison suggests that the weak $227 \mathrm{GHz}$ emission detected at PdBI arises from the

1 We measure a peak $98 \mathrm{GHz}$ flux density of $0.6 \mathrm{mJy} / 4.5^{\prime \prime}$-beam close to $\left(\sim 4^{\prime \prime}\right.$ north-east of) the central $227 \mathrm{GHz}$ position but the cleaned image contains negative contours down to $-0.8 \mathrm{mJy} / 4.5^{\prime \prime}$ beam. 


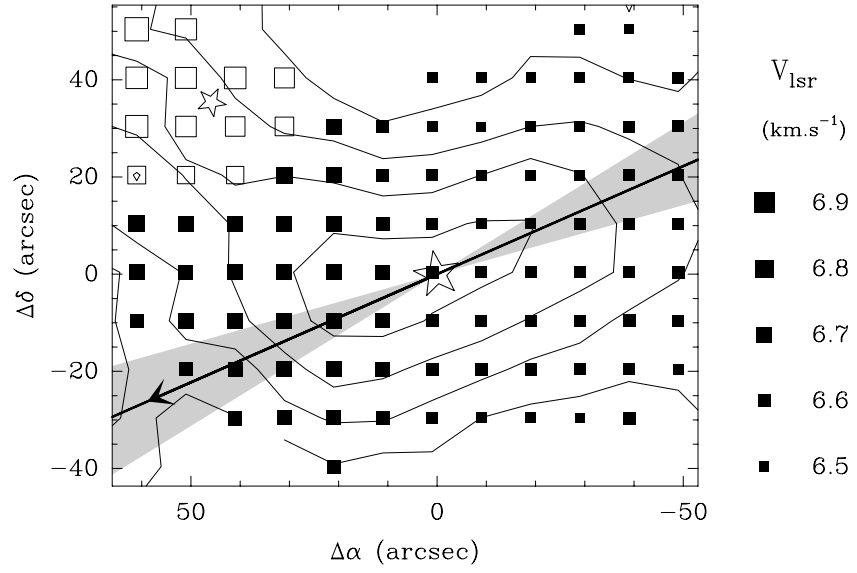

Fig. 2. Map of the peak LSR velocity (filled squares) in the IRAM 04191 envelope as derived from Gaussian fits to the $\mathrm{C}_{3} \mathrm{H}_{2}\left(2_{12}-\right.$ $1_{01}$ ) spectra observed with the $30 \mathrm{~m}$ telescope. The map of integrated $\mathrm{C}_{3} \mathrm{H}_{2}\left(2_{12}-1_{01}\right)$ intensity between 5.7 and $7.7 \mathrm{~km} \mathrm{~s}^{-1}$ is overlaid as contours (ranging from 0.4 to 1.0 by $0.2 \mathrm{~K} \mathrm{~km} \mathrm{~s}^{-1}$ ). The black arrow shows the best-fit direction ( $\mathrm{PA} \sim 114^{\circ} \pm 8^{\circ}$ ) of the mean velocity gradient $\left(\sim 3.4 \mathrm{~km} \mathrm{~s}^{-1} \mathrm{pc}^{-1}\right)$ across the envelope; the grey-shaded sectors display the $1 \sigma$ error on this orientation. The size of each square is proportional to the LSR velocity at its position. The open squares in the upper-left corner give the LSR velocities measured at positions that were ignored in fitting the velocity gradient because of likely contamination by the Class I source IRAS 04191 and/or the redshifted lobe of the IRAM 04191 outflow. The two star symbols mark the positions of IRAM 04191 (at $(0,0)$ ) and IRAS 04191.

inner part of the envelope seen at the $30 \mathrm{~m}$ telescope rather than from an accretion disk surrounding the central protostellar object. Furthermore, a single power law $I(\theta) \propto \theta^{-0.5 \pm 0.2}$ appears to characterize the radial intensity profile of the envelope over the whole range of angular radii from $\sim 1^{\prime \prime}$ to $\sim 100^{\prime \prime}$. Such an intensity profile corresponds to a $\rho \propto r^{-1.5}$ density profile if the dust temperature is uniform.

The weak emission detected at PdBI sets strong constraints on the mass and size of any central accretion disk. Assuming optically thin emission, a dust mass opacity of $0.02 \mathrm{~cm}^{2} \mathrm{~g}^{-1}$ typical of circumstellar disks (cf. Beckwith et al. 1990) and a mean dust temperature of $20 \mathrm{~K}$ in the disk, the PdBI peak flux density yields $M_{\text {disk }}<1 \times 10^{-3} M_{\odot}$. Alternatively, the assumption of optically thick dust emission at $20 \mathrm{~K}$ implies $R_{\text {disk }}<10 \mathrm{AU}$.

\subsection{Fast, differential rotation}

All of the centroid velocity (first-order moment) maps taken at the $30 \mathrm{~m}$ telescope in small optical depth lines, such as $\mathrm{C}_{3} \mathrm{H}_{2}\left(2_{12}-1_{01}\right), \mathrm{N}_{2} \mathrm{H}^{+}(101-012), \mathrm{H}^{13} \mathrm{CO}^{+}(1-0)$, and $\mathrm{C}^{34} \mathrm{~S}(2-$ 1 ), show a clear velocity gradient across the envelope (see, e.g., Fig. 2). The (south)-east part is redshifted with respect to the source systemic velocity, while the (north)-west part is blueshifted. We have applied the sector method described by Arquilla \& Goldsmith (1986) to measure the direction of this velocity gradient in the $\mathrm{C}_{3} \mathrm{H}_{2}\left(2_{12}-1_{01}\right)$ map using Gaussian fits

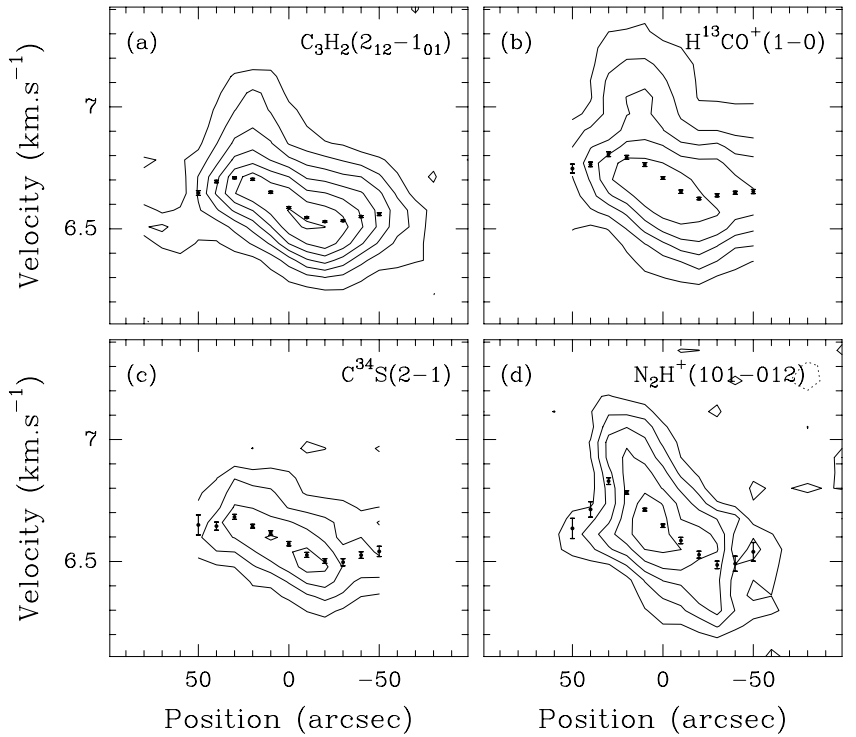

Fig. 3. Position-velocity diagrams in small optical depth lines along the direction perpendicular to the outflow axis. a) $\mathrm{C}_{3} \mathrm{H}_{2}\left(2_{12}-1_{01}\right)$; b) $\mathrm{H}^{13} \mathrm{CO}^{+}(1-0)$; c) $\mathrm{C}^{34} \mathrm{~S}(2-1)$; d) $\mathrm{N}_{2} \mathrm{H}^{+}(101-012)$. The dots with error bars represent the peak velocities as determined by Gaussian fits. The base contour and contour step have the same value: $0.3 \mathrm{~K}$ (in $T_{\mathrm{A}}^{*}$ ) in a) and b); $0.2 \mathrm{~K}$ in c) and d). Note how the dots mark a similar "S" shape in all four diagrams, suggestive of differential rotation in the envelope (see text).

to the spectra. We obtain a position angle $\mathrm{PA} \sim 114^{\circ} \pm 8^{\circ}$ (cf. Fig. 2). The velocity gradient thus lies along the major axis of the elongated dust $/ \mathrm{N}_{2} \mathrm{H}^{+}$core $\left(\mathrm{PA} \sim 120^{\circ}\right.$ ), i.e., perpendicular to the outflow axis. Coupled to the high degree of symmetry of the position-velocity diagrams shown in Fig. 3 with respect to envelope center, this strongly suggests that the envelope is rotating about an axis coinciding with the outflow axis (cf. Fig. 1). Turbulent motions would produce a more random velocity field (cf. Burkert \& Bodenheimer 2000) and are weak here anyway (see Sect. 3.4 and Fig. 12b below).

From now on, we adopt a position angle PA $=28^{\circ}$ for the projection of the rotation/outflow axis onto the plane of the sky. To minimize contamination by the outflow, we analyze the velocity structure of the envelope along the axis perpendicular to the outflow (i.e. along PA $=118^{\circ}$ ) and going through the center. Along this axis, the magnitude of the velocity gradient estimated from linear fits to the $\mathrm{C}_{3} \mathrm{H}_{2}\left(2_{12}-1_{01}\right), \mathrm{H}^{13} \mathrm{CO}^{+}(1-0), \mathrm{C}^{34} \mathrm{~S}(2-1)$, and $\mathrm{N}_{2} \mathrm{H}^{+}(101-012)$ position-velocity diagrams is $\sim 6 \mathrm{~km} \mathrm{~s}^{-1} \mathrm{pc}^{-1}, \sim 7 \mathrm{~km} \mathrm{~s}^{-1} \mathrm{pc}^{-1}$, $\sim 6 \mathrm{~km} \mathrm{~s}^{-1} \mathrm{pc}^{-1}$, and $10 \mathrm{~km} \mathrm{~s}^{-1} \mathrm{pc}^{-1}$, respectively, increasing from north-west to south-east over $\pm 20^{\prime \prime}$ (see Fig. 3). The mean velocity gradient is thus $7 \pm 2 \mathrm{~km} \mathrm{~s}^{-1} \mathrm{pc}^{-1}$ in the $2800 \mathrm{AU}$ radius region.

The $\mathrm{C}_{3} \mathrm{H}_{2}\left(2_{12}-1_{01}\right), \mathrm{H}^{13} \mathrm{CO}^{+}(1-0)$, and $\mathrm{N}_{2} \mathrm{H}^{+}(101-012)$ position-velocity diagrams shown in Fig. 3 may be slightly

2 Before fitting the direction of the velocity gradient, we have masked the redshifted emission seen in the north-east part of the $\mathrm{C}_{3} \mathrm{H}_{2}\left(2_{12}-1_{01}\right)$ map shown in Fig. 2, as it is likely associated with the redshifted lobe of the outflow or with the nearby Class I source IRAS 04191+1523 (Tamura et al. 1991). 

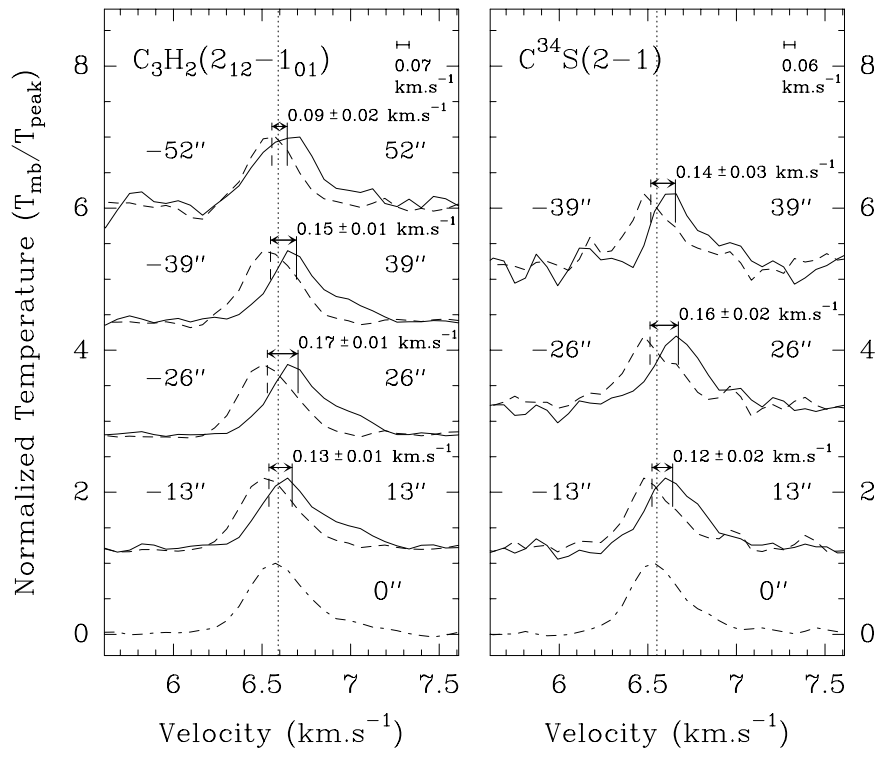

Fig. 4. Normalized $\mathrm{C}_{3} \mathrm{H}_{2}\left(2_{12}-1_{01}\right)$ and $\mathrm{C}^{34} \mathrm{~S}(2-1)$ spectra observed every half beamwidth along the direction perpendicular to the outflow axis. Each spectrum has been divided by its peak temperature for easier inter-comparison. In both panels, the vertical dotted line marks the peak velocity of the central spectrum (dash-dotted curve at the bottom) measured by a Gaussian fit. The double-sided arrows and associated values indicate the magnitude of the velocity shifts (determined by Gaussian fits) between spectra observed at symmetric positions $\left( \pm 13^{\prime \prime}, \pm 26^{\prime \prime}, \pm 39^{\prime \prime}\right.$, or $\left.\pm 52^{\prime \prime}\right)$ about the center. The channel resolution is shown in the upper right corner of each panel. While solid-body rotation would produce a velocity shift increasing linearly with radius, the opposite trend is observed beyond one beamwidth (26") from the center: the velocity shift decreases for larger offsets about the central position. This strongly suggests the presence of differential rotation in the envelope.

contaminated by a secondary, redshifted component toward the east. We have used the GAUSSCLUMPS algorithm of Stutzki \& Güsten (1990) (see also Kramer et al. 1998) to try and subtract this secondary component from the data cubes. The algorithm finds a Gaussian component at approximately $\left(+50^{\prime \prime}\right.$, $\left.+30^{\prime \prime}\right)$ in $\mathrm{C}_{3} \mathrm{H}_{2}\left(2_{12}-1_{01}\right)$ and $\left(+30^{\prime \prime},+20^{\prime \prime}\right)$ in $\mathrm{H}^{13} \mathrm{CO}^{+}(1-0)$. The removal of this secondary component reduces the magnitude of the velocity gradient measured in $\mathrm{C}_{3} \mathrm{H}_{2}\left(2_{12}-1_{01}\right)$ and $\mathrm{H}^{13} \mathrm{CO}^{+}(1-0)$ by $\sim 30 \%$. GAUSSCLUMPS fails to identify any secondary component near the same location in the $\mathrm{N}_{2} \mathrm{H}^{+}(101-$ $012)$ data cube. The velocity gradient thus seems to be significantly larger in $\mathrm{N}_{2} \mathrm{H}^{+}(101-012)$ than in the other three lines (see below).

Remarkably, all of the position-velocity diagrams shown here present a "S" shape, clearly seen on the peak velocity curves derived from Gaussian fits (see Fig. 3). On either side of the source, the velocity shift with respect to the systemic velocity increases in absolute value up to an angular radius $\theta_{\mathrm{m}} \sim 25 \pm 10^{\prime \prime}$ (i.e., one full beamwidth of the $30 \mathrm{~m}$ telescope at $3 \mathrm{~mm}$, corresponding to $\left.r_{\mathrm{m}} \sim 3500 \pm 1400 \mathrm{AU}\right)$, and then decreases for $\theta>\theta_{\mathrm{m}}$. This is further illustrated in Fig. 4 which shows the $\mathrm{C}_{3} \mathrm{H}_{2}\left(2_{12}-1_{01}\right)$ and $\mathrm{C}^{34} \mathrm{~S}(2-1)$ spectra observed every half beamwidth (Nyquist sampling) at symmetric positions with respect to source center along the direction perpendicular to the outflow axis. It can be seen that the velocity shift between symmetric positions decreases from $0.17 \mathrm{~km} \mathrm{~s}^{-1}$ (i.e., 2.5 channels) at $\pm 26^{\prime \prime}$ to $0.09 \mathrm{~km} \mathrm{~s}^{-1}$ (1.3 ch.) at $\pm 52^{\prime \prime}$ in $\mathrm{C}_{3} \mathrm{H}_{2}\left(2_{12}-1_{01}\right)$. In $\mathrm{C}^{34} \mathrm{~S}(2-1)$, the velocity shift varies from $0.16 \mathrm{~km} \mathrm{~s}^{-1}$ (2.6 ch.) at $\pm 26^{\prime \prime}$ to $0.14 \mathrm{~km} \mathrm{~s}^{-1}$ (2.3 ch.) at $\pm 39^{\prime \prime}$. These values translate into a decrease of the $\mathrm{C}_{3} \mathrm{H}_{2}\left(2_{12}-1_{01}\right)$ velocity gradient from $4.8 \mathrm{~km} \mathrm{~s}^{-1} \mathrm{pc}^{-1}$ to $1.3 \mathrm{~km} \mathrm{~s}^{-1} \mathrm{pc}^{-1}$ between $26^{\prime \prime}$ and $52^{\prime \prime}$, and to a decrease of the $\mathrm{C}^{34} \mathrm{~S}(2-1)$ velocity gradient from $4.5 \mathrm{~km} \mathrm{~s}^{-1} \mathrm{pc}^{-1}$ to $2.6 \mathrm{~km} \mathrm{~s}^{-1} \mathrm{pc}^{-1}$ between $26^{\prime \prime}$ and $39^{\prime \prime}$. The decrease of the velocity shift with radius beyond $\pm 26^{\prime \prime}$ is in marked contrast with the linear increase expected in the case of solid-body rotation. We thus conclude that there is strong evidence for differential rotation in the envelope beyond $\sim 3500$ AU.

Correcting for inclination $\left(i=50^{\circ}\right.$, cf. Sect. 1.2) and taking all four lines of Fig. 3 into account, we estimate that the rotational angular velocity is $\Omega=9 \pm 3 \mathrm{~km} \mathrm{~s}^{-1} \mathrm{pc}^{-1}$ $\left(\sim 3 \times 10^{-13} \mathrm{rads}^{-1}\right)$ at $r=2800 \mathrm{AU}\left(20^{\prime \prime}\right)^{3}$ and a factor of $\sim 5$ lower, i.e., $\Omega=1.9 \pm 0.2 \mathrm{~km} \mathrm{~s}^{-1} \mathrm{pc}^{-1}$, at $r=7000 \mathrm{AU}$ $\left(50^{\prime \prime}\right)$.

At a radius $r \sim 11000 \mathrm{AU}$, our $\mathrm{C}^{18} \mathrm{O}(1-0)$ and $\mathrm{C}^{18} \mathrm{O}(2-$ 1) observations suggest an even smaller angular velocity, $\Omega \lesssim 0.5-1 \mathrm{~km} \mathrm{~s}^{-1} \mathrm{pc}^{-1}\left(\sim 1.5-3 \times 10^{-14} \mathrm{rad} \mathrm{s}^{-1}\right)$. On scales smaller than the beam, the intrinsic rotation velocity pattern is uncertain due to insufficient spatial resolution (the beam HWHM angular radius is $\sim 13^{\prime \prime}$ at $3 \mathrm{~mm}$, corresponding to a physical radius of $\sim 1800 \mathrm{AU})$. However, two indirect arguments suggest that the differential rotation pattern observed here between $\sim 3500$ AU and $\sim 11000$ AU continues down to smaller ( 1000-2000 AU) scales. First, such a differential rotation pattern, combined with a lower level of molecular depletion near envelope center in $\mathrm{N}_{2} \mathrm{H}^{+}$(cf. Sects. 3.4 and 4.2 below), would explain the higher velocity gradient $\left(10 \mathrm{~km} \mathrm{~s}^{-1} \mathrm{pc}^{-1}\right)$ measured in $\mathrm{N}_{2} \mathrm{H}^{+}(101-012)$ over $\pm 20^{\prime \prime}$ compared to the other three lines shown in Fig. 3. Second, using $\mathrm{NH}_{3}$ interferometric observations sensitive to $\sim 5^{\prime \prime}-15^{\prime \prime}$ scales, Wootten et al. (2001) have recently reported an even larger gradient $\left(\sim 15 \mathrm{~km} \mathrm{~s}^{-1} \mathrm{pc}^{-1}\right)$ than our present $\mathrm{N}_{2} \mathrm{H}^{+}$value.

\subsection{Spectroscopic signature of collapse}

As already pointed out by AMB99, the classical spectroscopic signature of infall motions (cf. Evans 1999; Myers et al. 2000) is seen toward IRAM 04191. Optically thick lines such as $\mathrm{CS}(2-1), \mathrm{CS}(3-2), \mathrm{CS}(5-4), \mathrm{H}_{2} \mathrm{CO}\left(2_{12}-1_{11}\right)$ and $\mathrm{H}_{2} \mathrm{CO}\left(3_{12}-\right.$ $2_{11}$ ) are double-peaked and skewed to the blue, while low optical depth lines such as $C^{34} S(2-1)$ and $C^{34} S(3-2)$ peak in the dip of the self-absorbed lines (see spectra observed at the central position in Fig. 5). Blue-skewed CS(2-1) and CS(3-2) spectra are observed in an extended region, up to an angular radius of at least $40^{\prime \prime}$ (5600 $\mathrm{AU}$ ) from source center (see cut taken

${ }^{3}$ With such an angular velocity, the gas at $r \sim 2800$ AU would make a complete turn in $\sim 0.7 \mathrm{Myr}$, which is comparable to the typical lifetime of prestellar cores with central densities $\gtrsim 10^{5} \mathrm{~cm}^{-3}$ (e.g. Jessop \& Ward-Thompson 2000). Thus, although the observed velocity gradient is probably not indicative of a well-developed circular motion, it may have already induced significant rotational distortion in the envelope. 

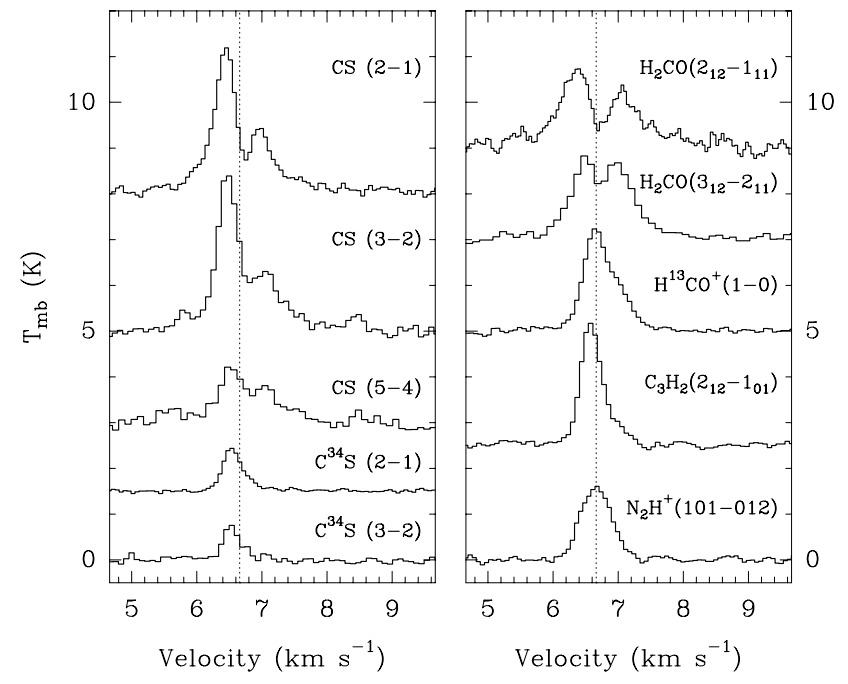

Fig. 5. Spectral line profiles observed at the central position of IRAM 04191 in the optically thick CS(2-1), CS(3-2), CS(5-4), $\mathrm{H}_{2} \mathrm{CO}\left(2_{12}-1_{11}\right), \mathrm{H}_{2} \mathrm{CO}\left(3_{12}-2_{11}\right)$ lines, and the low optical depth $\mathrm{C}^{34} \mathrm{~S}(2-1), \mathrm{C}^{34} \mathrm{~S}(3-2), \mathrm{H}^{13} \mathrm{CO}^{+}(1-0), \mathrm{C}_{3} \mathrm{H}_{2}\left(2_{12}-1_{01}\right), \mathrm{N}_{2} \mathrm{H}^{+}(101-012)$ lines. The dotted line indicates the source systemic velocity estimated from a seven-component hyperfine structure fit to the $\mathrm{N}_{2} \mathrm{H}^{+}(1-0)$ multiplet.

perpendicular to the outflow axis in Fig. 8 below). Such asymmetric line profiles with a blue peak stronger than the red peak are expected in a collapsing envelope when the line excitation temperature increases toward the center. There is therefore strong evidence for the presence of extended inward motions in the IRAM 04191 envelope.

The blue-to-red peak intensity ratio of the CS lines is weaker toward the south-east (i.e. the envelope hemisphere redshifted by rotation), while the asymmetry is stronger toward the north-west (i.e. the hemisphere blue-shifted by rotation) where the red peak is even barely visible. This behavior is in qualitative agreement with the expected distortion of the infall asymmetry due to rotation when the rotation velocity does not dominate over the infall velocity ${ }^{4}$ (Zhou 1995).

The dips of the optically thick $\mathrm{CS}(2-1), \mathrm{CS}(3-2)$, and $\mathrm{CS}(5-4)$ lines have velocities of $6.75 \pm 0.03 \mathrm{~km} \mathrm{~s}^{-1}, 6.81 \pm$ $0.04 \mathrm{~km} \mathrm{~s}^{-1}$, and $6.86 \pm 0.05 \mathrm{~km} \mathrm{~s}^{-1}$, respectively, and are redshifted relative to the source systemic velocity of $6.66 \pm$ $0.03 \mathrm{~km} \mathrm{~s}^{-1}$ (see Fig. 5). The latter value results from a 7component Gaussian fit to the $\mathrm{N}_{2} \mathrm{H}^{+}(1-0)$ multiplet, using the hyperfine structure (hfs) method of the CLASS reduction software, and assuming the relative frequencies and intensities of the 7 hyperfine components determined by Caselli et al. (1995), and the $\mathrm{N}_{2} \mathrm{H}^{+}$(101-012) rest frequency of Lee et al. (2001) (see Table 1). The uncertainty on the Lee et al. (2001) frequency is estimated to be $\sim 0.02 \mathrm{~km} \mathrm{~s}^{-1}$, to which we conservatively add (in quadrature) an uncertainty of $0.015 \mathrm{~km} \mathrm{~s}^{-1}$ arising from a maximum pointing error of $\sim 5^{\prime \prime}$, given the velocity gradient discussed in Sect. 3.2 above. This yields a final uncertainty of $\sim 0.03 \mathrm{~km} \mathrm{~s}^{-1}$ on the source systemic velocity. The dips of the

\footnotetext{
${ }^{4}$ When the rotation velocity becomes comparable to the infall velocity, the asymmetry may even be reversed (e.g. Walker et al. 1994; Ward-Thompson \& Buckley 2001).
}

self-absorbed CS(2-1), CS(3-2), and CS(5-4) spectra are thus redshifted relative to the systemic velocity by $0.09 \pm 0.04 \mathrm{~m} \mathrm{~s}^{-1}$, $0.15 \pm 0.05 \mathrm{~km} \mathrm{~s}^{-1}$, and $0.20 \pm 0.06 \mathrm{~km} \mathrm{~s}^{-1}$, respectively. These absorption dips are presumably produced by the outer layers of the front hemisphere of the envelope. The fact that they are redshifted provides a second indication that inward motions are present in the outer envelope where the opacity of the CS lines is of order unity. More quantitatively, the main-beam brightness temperature of the dip in the central $\mathrm{CS}(2-1)$ spectrum is $\sim 0.7 \mathrm{~K}$, which matches the peak temperature of the spectra taken at $\sim 70^{\prime \prime}$ from source center. These spectra are still optically thick since we measure a $\mathrm{CS}(2-1)$ to $\mathrm{C}^{34} \mathrm{~S}(2-1)$ integrated intensity ratio of only $\sim 5$, i.e., $\sim 4$ times less than the standard CS to $\mathrm{C}^{34} \mathrm{~S}$ isotopic ratio of $\sim 22$ (Wilson \& Rood 1994). Assuming a spherically symmetric envelope, we conclude that the absorbing shell producing the dip in the central $\mathrm{CS}(2-1)$ spectrum has an angular radius larger than $\sim 70^{\prime \prime}$. The observed $\sim 0.1 \mathrm{~km} \mathrm{~s}^{-1}$ redshift of the dip is thus suggestive of inward motions $\sim 0.1 \mathrm{~km} \mathrm{~s}^{-1}$ extending up to a radius of at least 10000 AU. Radiative transfer simulations confirm this conclusion (see Sect. 4 below).

On the other hand, the $\mathrm{CS}(5-4)$ emission is much more concentrated spatially than the $\mathrm{CS}(2-1)$ emission, and confined to the inner $20^{\prime \prime}$ (FWHM) region (see the non-detection at $10^{\prime \prime}$ in Fig. 8 below). The radius of the shell producing the absorption dip in the central $\mathrm{CS}(5-4)$ spectrum must therefore be smaller than $10^{\prime \prime}$. The observed $\sim 0.2 \mathrm{~km} \mathrm{~s}^{-1}$ redshift of the CS(5-4) dip then suggests faster inward motions in the central $(r<1400 \mathrm{AU})$ region.

\subsection{Linewidths: Evidence for turbulent infall?}

The $C^{34} S(2-1)$ and $C^{34} S(3-2)$ spectra displayed in Fig. 5 are slightly asymmetric and skewed to the blue, which suggests they are marginally optically thick and showing some infall asymmetry (see, e.g., Fig. 1 of Myers et al. 1995). We measure $(F W H M)$ linewidths of $0.38 \pm 0.01 \mathrm{~km} \mathrm{~s}^{-1}$ for $\mathrm{C}^{34} \mathrm{~S}(2-$ 1) and $0.32 \pm 0.03 \mathrm{~km} \mathrm{~s}^{-1}$ for $\mathrm{C}^{34} \mathrm{~S}(3-2)$, which are 3.8 and 3.2 times larger than the thermal broadening of $\mathrm{C}^{34} \mathrm{~S}$ at a kinetic temperature $T_{\mathrm{K}}=10 \mathrm{~K}$, respectively (see Sect. 4.1 for constraints on $T_{\mathrm{K}}$ ). Radiative transfer simulations (cf. Sect. 4) indicate opacities of $\sim 1.5$ and $\sim 1$ for $C^{34} S(2-1)$ and $C^{34} S(3-2)$, respectively. Therefore, line saturation effects cannot broaden the $\mathrm{C}^{34} \mathrm{~S}(2-1)$ and $\mathrm{C}^{34} \mathrm{~S}(3-2)$ spectra by more than $\sim 30 \%$ and $\sim 20 \%$, respectively, and the linewidths are primarily nonthermal. Motions such as infall, rotation, outflow, or "turbulence" along the line of sight are required to explain such nonthermal linewidths. The nonthermal motions do not dominate over thermal motions, however, since the $C^{34} S(2-1)$ and $C^{34} S(3-2)$ linewidths represent only $\sim 80 \%$ of the thermal velocity dispersion for a mean particle of molecular weight $\mu=2.33$. The $\mathrm{N}_{2} \mathrm{H}^{+}(101-012)$ line is slightly broader, with a $F W H M \sim$ $0.55 \mathrm{~km} \mathrm{~s}^{-1}$, i.e., 1.2 times broader than the (mean particle) thermal velocity dispersion and $\sim 1.5$ times larger than the $\mathrm{C}^{34} \mathrm{~S}$ linewidths. The hyperfine structure fit to the $\mathrm{N}_{2} \mathrm{H}^{+}$multiplet (see Sect. 3.3) yields an optical depth of $\sim 0.85$ for the isolated

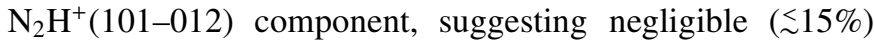


optical depth broadening. The level of optical depth broadening should thus be more pronounced in $\mathrm{C}^{34} \mathrm{~S}$ and cannot explain the difference in linewidth between $\mathrm{N}_{2} \mathrm{H}^{+}$and $\mathrm{C}^{34} \mathrm{~S}$.

We propose that this difference in linewidth between $\mathrm{N}_{2} \mathrm{H}^{+}$ and $\mathrm{C}^{34} \mathrm{~S}$ results from a combination of higher infall/rotation velocities and lower $\mathrm{N}_{2} \mathrm{H}^{+}$depletion toward the center. Bergin \& Langer (1997) have shown that sulphur-bearing molecules such as CS are strongly depleted when the density increases, whereas $\mathrm{N}_{2} \mathrm{H}^{+}$remains in the gas phase, at least up to densities $n_{\mathrm{H}_{2}} \lesssim 10^{6} \mathrm{~cm}^{-3}$. Indeed, we measure a decrease of the $\mathrm{C}^{34} \mathrm{~S}(2-$ 1)/ $\mathrm{N}_{2} \mathrm{H}^{+}(101-012)$ integrated intensity ratio by a factor of 2 from $\sim 5000 \mathrm{AU}$ to $\sim 2000 \mathrm{AU}$. As both lines have nearly the same critical density and are approximately optically thin ${ }^{5}$, the decrease of the integrated intensity ratio may be interpreted as a decrease of the $\mathrm{C}^{34} \mathrm{~S} / \mathrm{N}_{2} \mathrm{H}^{+}$abundance ratio toward the center.

Finally, we note that the $\mathrm{N}_{2} \mathrm{H}^{+}(101-012)$ linewidth peaks at the central position (as shown by a linewidth-position plot along the direction perpendicular to the ouflow axis). $\mathrm{N}_{2} \mathrm{H}^{+}$ may thus be more sensitive to higher velocity material produced by, e.g., infall, rotation, or outflow near the central protostellar object. As $\mathrm{N}_{2} \mathrm{H}^{+}$is generally underabundant in molecular outflows (e.g. Bachiller \& Pérez-Gutiérrez 1997), the central broadening of $\mathrm{N}_{2} \mathrm{H}^{+}(1-0)$ is most likely due to infall and/or rotation motions.

\subsection{The CS line wing emission}

The morphology of the single-dish CS(2-1) maps integrated over the $4.6-6.1 \mathrm{~km} \mathrm{~s}^{-1}$ (blue) and 7.1-8.6 $\mathrm{km} \mathrm{s}^{-1}$ (red) velocity ranges strongly suggests that the CS line wing emission is dominated by material associated with the outflow (see Figs. 6a and b). Some CS(2-1) emission is detected at the edges of both the red and the blue lobe of the CO outflow: the $\mathrm{CS}(2-1)$ red wing is relatively weak and concentrated at the south-eastern edge and the tip of the red CO lobe (Fig. 6a), while the CS(21) blue wing is much stronger and distributed in two spots on either side of the blue CO lobe (Fig. 6b). This "high-velocity" $\mathrm{CS}(2-1)$ emission is likely to arise from dense, shocked material entrained by the outflow.

The redshifted CS(2-1) emission detected on smaller scales by the PdBI interferometer in the $7-8 \mathrm{~km} \mathrm{~s}^{-1}$ velocity range also appears to be associated with the outflow. This emission arises from the base of the red CO lobe and its shape closely follows the edges of the outflow lobe (see Fig. 6c). No emission was detected by PdBI in the blue wing range (see Fig. 6d) or at the source systemic velocity. The blueshifted CS(2-1) emission seen in the single-dish map (Fig. 6b) thus appears to be more extended than the redshifted emission and is likely resolved out by the interferometer. Such a difference in spatial extent between blueshifted and redshifted emission, opposite of what infall motions would produce, is expected if the CS line wing emission arises from outflowing material. Likewise, the emission detected by the $30 \mathrm{~m}$ telescope near the systemic velocity, i.e., close to the dip of the $\mathrm{CS}(2-1)$ line (Fig. 5), arises from

\footnotetext{
${ }^{5}$ Based on the opacities derived above, optical depth effects cannot account for more than a factor of 1.3 decrease in the $\mathrm{C}^{34} \mathrm{~S}(2-$ 1)/ $\mathrm{N}_{2} \mathrm{H}^{+}(101-012)$ ratio.
}
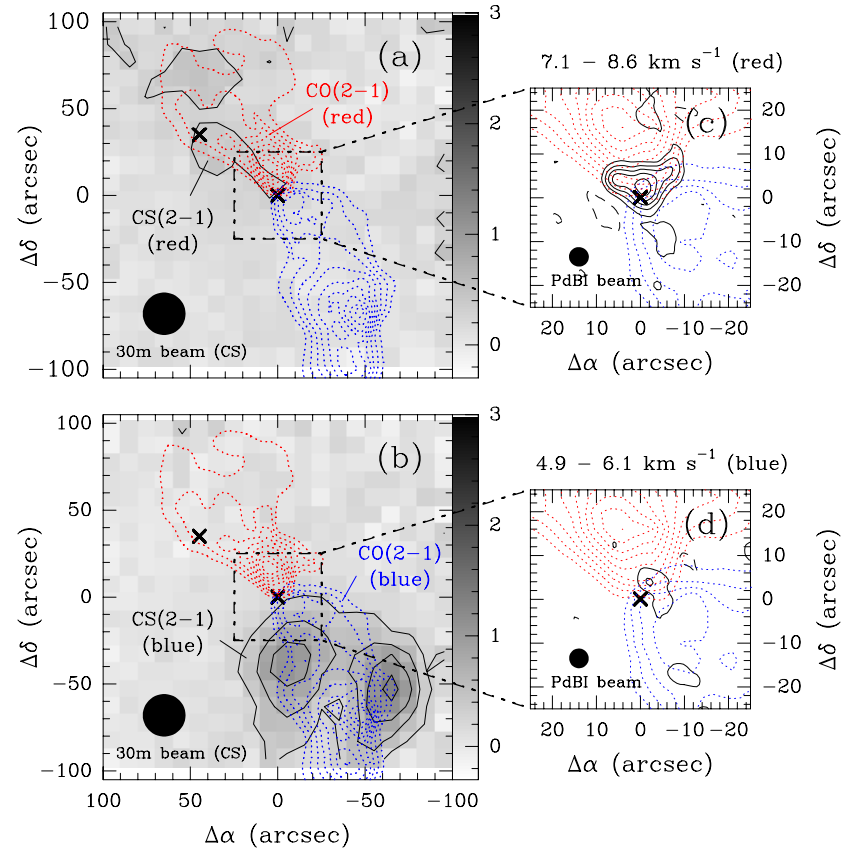

Fig. 6. $\mathrm{CS}(2-1)$ integrated intensity maps taken with the $30 \mathrm{~m}$ telescope (left) and, on a smaller scale, with the PdBI interferometer (right). a) Single-dish map integrated in the 7.1 to $8.6 \mathrm{~km} \mathrm{~s}^{-1}$ velocity range (red), overlaid on the $\mathrm{CO}(2-1)$ outflow contours from AMB99. b) Same as a) for the 4.6 to $6.1 \mathrm{~km} \mathrm{~s}^{-1}$ velocity range (blue). The CS wing emission (solid contours and gray scale) appears to be correlated with the $\mathrm{CO}$ outflow emission (dotted contours). The two crosses mark the central positions of IRAM $04191($ at $(0,0))$ and IRAS 04191. The $\mathrm{CS}(2-1)$ contour step is $0.3 \mathrm{~K} \mathrm{~km} \mathrm{~s}^{-1}$; the gray scale (shown on the right of each map) ranges from -0.3 to $3 \mathrm{~K} \mathrm{~km} \mathrm{~s}^{-1}$. c) Cleaned interferometer map, integrated over the 7.1 to $8.6 \mathrm{~km} \mathrm{~s}^{-1}$ velocity range (red), overlaid on the $\mathrm{CO}(2-1)$ contours of the outflow. d) Same as c) for the 4.9 to $6.1 \mathrm{~km} \mathrm{~s}^{-1}$ velocity range (blue). The CS(2-1) contour step is $0.25 \mathrm{Jy}_{\text {beam }}^{-1} \mathrm{~km} \mathrm{~s}^{-1}$. The clean beam is shown on the bottom left. Note how the CS(2-1) red wing emission detected on a $\sim 5^{\prime \prime}$ scale coincides with the base of the red lobe of the $\mathrm{CO}$ outflow, while the CS(2-1) blue wing emission is almost entirely resolved out by the interferometer.

extended foreground material on scales $\sim 70^{\prime \prime}$ (see Sect. 3.3) and is also resolved out by PdBI.

\subsection{Low degree of ionization}

Following, e.g., Caselli et al. (1998), we can estimate the degree of ionization in the IRAM 04191 envelope from the observed value of the abundance ratio $R_{\mathrm{D}}=\left[\mathrm{DCO}^{+}\right] /\left[\mathrm{HCO}^{+}\right]$. We measure an integrated intensity ratio $I_{\mathrm{DCO}^{+}} / I_{\mathrm{H}^{13} \mathrm{CO}^{+}}=3.3 \pm$ 0.6 at the central position. Assuming optically thin $\mathrm{H}^{13} \mathrm{CO}^{+}(3-$ 2) and $\mathrm{DCO}^{+}(3-2)$ emission, this implies $\left[\mathrm{DCO}^{+}\right] /\left[\mathrm{H}^{13} \mathrm{CO}^{+}\right]=$ $3.3 \pm 0.6$. Adopting a $\left[{ }^{12} \mathrm{C}\right] /\left[{ }^{13} \mathrm{C}\right]$ abundance ratio of 77 in the local ISM (Wilson \& Rood 1994), we thus derive $R_{\mathrm{D}}=$ $0.04 \pm 0.01$, which is identical to the ratio measured by Caselli et al. (2002b) toward the central position of L1544. As the same density $\left(\sim 10^{6} \mathrm{~cm}^{-3}\right)$ is probed by the observations in both cases, we conclude that the ionization degree $x_{i} \sim 2 \times 10^{-9}$ derived in L1544 by Caselli et al. (2002b) based on their chemical models should be representative of the ionization degree in the 

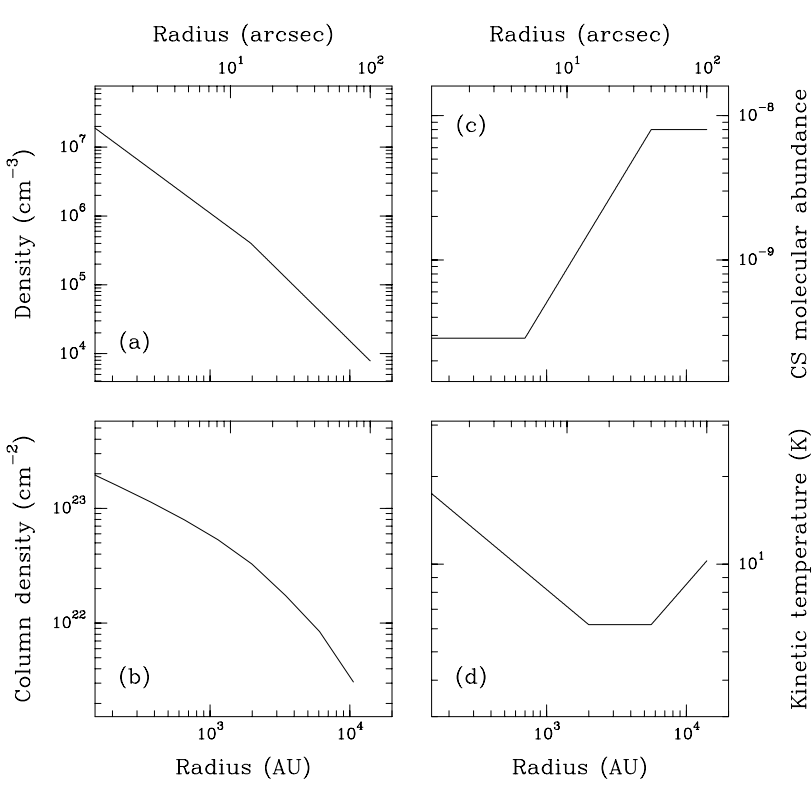

Fig. 7. Input physical properties of the 1D spherical envelope model used to fit the observed CS and $\mathrm{C}^{34} \mathrm{~S}$ spectra (see Fig. 8): Plots of density a), column density b), $\mathrm{CS} / \mathrm{H}_{2}$ relative abundance c), and kinetic temperature $\mathbf{d}$ ) as a function of radius.

IRAM 04191 envelope at this density (assuming similar depletion factors for CO, which seems likely - see Sect. 4.2 below).

\section{Radiative transfer modeling: 1D spherical simulations}

Here, we use the radiative transfer code MAPYSO (Blinder 1997) to model the observed line spectra and set quantitative constraints on the kinematics of the IRAM 04191 envelope (see Appendix for details about the code). For simplicity, we use a piecewise powerlaw description for the spatial variations of the kinetic temperature, density, molecular abundance, infall velocity, and rotational velocity (in Sect. 5 below) across the envelope (see Fig. 7).

\subsection{Model inputs: Mass distribution and kinetic temperature profile}

We use the envelope mass distribution derived by Motte \& André (2001) (hereafter MA01) from $1.3 \mathrm{~mm}$ dust continuum observations with the $30 \mathrm{~m}$ telescope. MA01 estimate that the envelope mass contained within a radius of $4200 \mathrm{AU}$ is $M_{\text {env }}(r<4200 \mathrm{AU})=0.45 M_{\odot}$ with an uncertainty of a factor of 2 on either side, assuming a mean dust temperature $T_{\text {dust }}=12.5 \pm 2.5 \mathrm{~K}$ obtained by AMB99 from a graybody fit to the $\lambda>90 \mu \mathrm{m}$ portion of the SED (see Fig. 3 of AMB99). In addition, the radial structure analysis of MA01 indicates an average radial intensity profile $I \propto \theta^{-m}$ with $m=0.6 \pm 0.1$ in the range of angular radii $\theta \sim 11^{\prime \prime}-100^{\prime \prime}$ (i.e., $r \sim 1500-14000 \mathrm{AU})$. Assuming a dust temperature profile $T_{\text {dust }} \propto r^{-q}$ with $q=-0.2 \pm 0.2$, MA01 obtain a density profile $\rho \propto r^{-p}$ with $p=m+1-q=1.8 \pm 0.3$. Given the low bolometric luminosity of IRAM 04191, the dust temperature is indeed expected to rise outward $(q<0)$ due to external heating by the interstellar radiation field (e.g. Masunaga \& Inutsuka 2000; Evans et al. 2001; Zucconi et al. 2001). The regime of central heating by the accreting protostar $(q \sim 0.4)$ is likely confined to the inner $\sim 2000 \mathrm{AU}$ radius region (cf. MA01). Since a good thermal coupling between gas and dust grains is expected for densities $n_{\mathrm{H}_{2}}>10^{5} \mathrm{~cm}^{-3}$ (e.g. Ceccarelli et al. 1996; Doty $\&$ Neufeld 1997), the gas kinetic temperature profile should track the dust temperature profile, at least up to a radius of $\sim 4000$ AU.

We also have some constraints on the gas kinetic temperature from our $\mathrm{C}^{18} \mathrm{O}, \mathrm{N}_{2} \mathrm{H}^{+}$, and $\mathrm{CS}$ observations. We measure a $\mathrm{C}^{18} \mathrm{O}(2-1)$ to $\mathrm{C}^{18} \mathrm{O}(1-0)$ integrated intensity ratio $I_{\mathrm{C}^{18} \mathrm{O}(2-1)} / I_{\mathrm{C}^{18} \mathrm{O}(1-0)}=1.5 \pm 0.3$ in the range of angular radii $0^{\prime \prime}-60^{\prime \prime}$, using main beam temperatures and after degrading the resolution of the $J=2-1$ data to that of the $J=1-0$ data. As the critical densities of $\mathrm{C}^{18} \mathrm{O}(1-0)$ and $\mathrm{C}^{18} \mathrm{O}(2-1)$ are $3 \times 10^{3} \mathrm{~cm}^{-3}$ and $2 \times 10^{4} \mathrm{~cm}^{-3}$ at $10 \mathrm{~K}$, respectively, both lines should be thermalized in most of the envelope. Assuming local thermodynamic equilibrium (LTE) and optically thin emission, we derive an excitation temperature $T_{\mathrm{ex}}\left(\mathrm{C}^{18} \mathrm{O}\right)=10 \pm 2 \mathrm{~K}$, which should be a good estimate of the gas kinetic temperature in the low-density outer $(r \sim 6000 \mathrm{AU})$ part of the envelope probed by $\mathrm{C}^{18} \mathrm{O}$. Likewise, the excitation temperature of the dense-gas tracer $\mathrm{N}_{2} \mathrm{H}^{+}(101-012)$ may be estimated from the relative intensities of the seven components of the $\mathrm{N}_{2} \mathrm{H}^{+}(1-0)$ multiplet, assuming the same excitation temperature for all components (cf. Sect. 3.3). This method yields $T_{\text {ex }}\left(\mathrm{N}_{2} \mathrm{H}^{+}\right)=5.5 \pm 0.5 \mathrm{~K}$. With a critical density $\sim 2 \times 10^{5} \mathrm{~cm}^{-3}$, the $\mathrm{N}_{2} \mathrm{H}^{+}(1-0)$ multiplet is probably thermalized only at radii $r<2000 \mathrm{AU}$. We thus obtain a lower limit of $\sim 6 \mathrm{~K}$ for the gas kinetic temperature in the $\mathrm{N}_{2} \mathrm{H}^{+}$-emitting part of the envelope ( $r \lesssim 5000 \mathrm{AU})$. Finally, the weak intensities of the optically thick $\mathrm{CS}(2-1)$ and $\mathrm{CS}(3-2)$ lines require a low gas kinetic temperature $\sim 6-7 \mathrm{~K}$ in the range of radii $2000-6000 \mathrm{AU}$ : Given the density profile shown in Fig. 7a, a uniform gas temperature of $10 \mathrm{~K}$ would produce $\mathrm{CS}(2-1)$ and $\mathrm{CS}(3-2)$ spectra with main beam temperatures about $1-2 \mathrm{~K}$ stronger than the observed temperatures. Beyond $r \sim 6000 \mathrm{AU}$, the gas temperature is likely to increase to the typical $\sim 10 \mathrm{~K}$ temperature of the Taurus cloud (see, e.g., Benson \& Myers 1989). However, the gas temperature profile in the outer parts of the envelope has little influence on the $\mathrm{CS}$ and $\mathrm{C}^{34} \mathrm{~S}$ spectra since the observed lines are far from LTE there.

In summary, the gas kinetic temperature profile is likely to present a minimum of $\sim 6 \mathrm{~K}$ at $\sim 10^{\prime \prime}-20^{\prime \prime}$ (i.e., $r \sim$ 1400-2800 AU) and to reach a value of $\sim 10 \mathrm{~K}$ in the outer parts of the envelope (cf. Fig. 7d).

\subsection{Molecular depletion}

Assuming a standard isotopic ratio $\chi_{\mathrm{CS}} / \chi_{\mathrm{C}^{34} \mathrm{~S}}=22$, a $[\mathrm{CS}] /\left[\mathrm{H}_{2}\right]$ abundance ratio of $8 \times 10^{-9}$ is required to match the $\mathrm{C}^{34} \mathrm{~S}(2-1)$ integrated intensity at an angular radius $40^{\prime \prime}-50^{\prime \prime}$. But a uniform abundance with such a value produces too strong $\mathrm{C}^{34} \mathrm{~S}(2-$ 1), $C^{34} S(3-2)$, and $C S(5-4)$ spectra toward the center. A good fit to the $\mathrm{C}^{34} \mathrm{~S}(2-1)$ integrated line intensities is obtained by assuming that the relative CS abundance drops by a factor of 


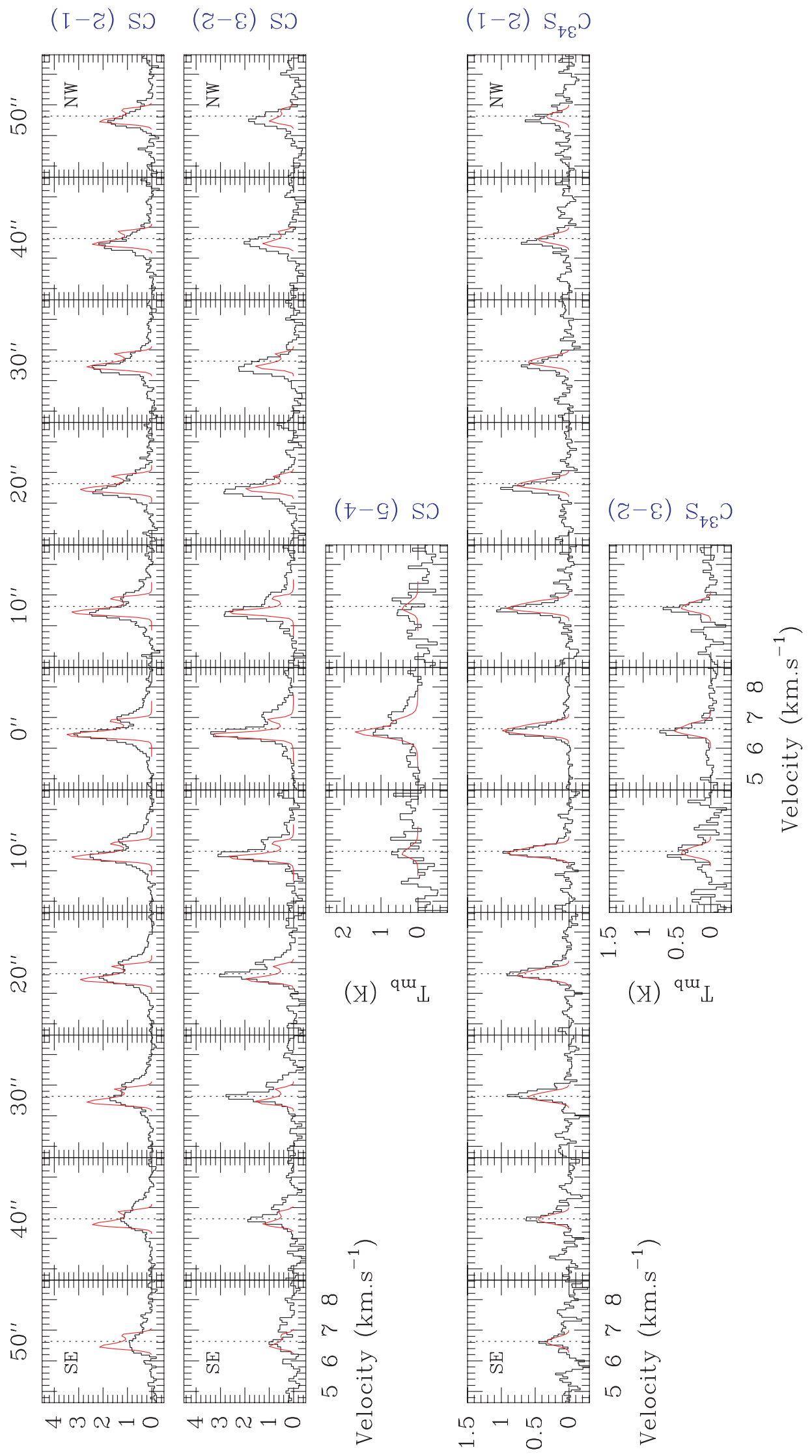

Fig. 8. $C S(2-1), C S(3-2), C S(5-4), C^{34} S(2-1)$, and $C^{34} S(3-2)$ spectra (in units of main beam temperature) observed along the direction perpendicular to the outflow axis (histograms). The dotted line indicates our best-fit estimate $\left(6.63 \mathrm{~km} \mathrm{~s}^{-1}\right)$ of the envelope systemic velocity based on our $\mathrm{CS} / \mathrm{C}^{34} \mathrm{~S}$ modeling. Synthetic spectra corresponding to the "best-fit" $1 \mathrm{D}$ spherical collapse model described in Sect. 4.3 (cf. Figs. 7 and 12a,b for model parameters) are superimposed. 


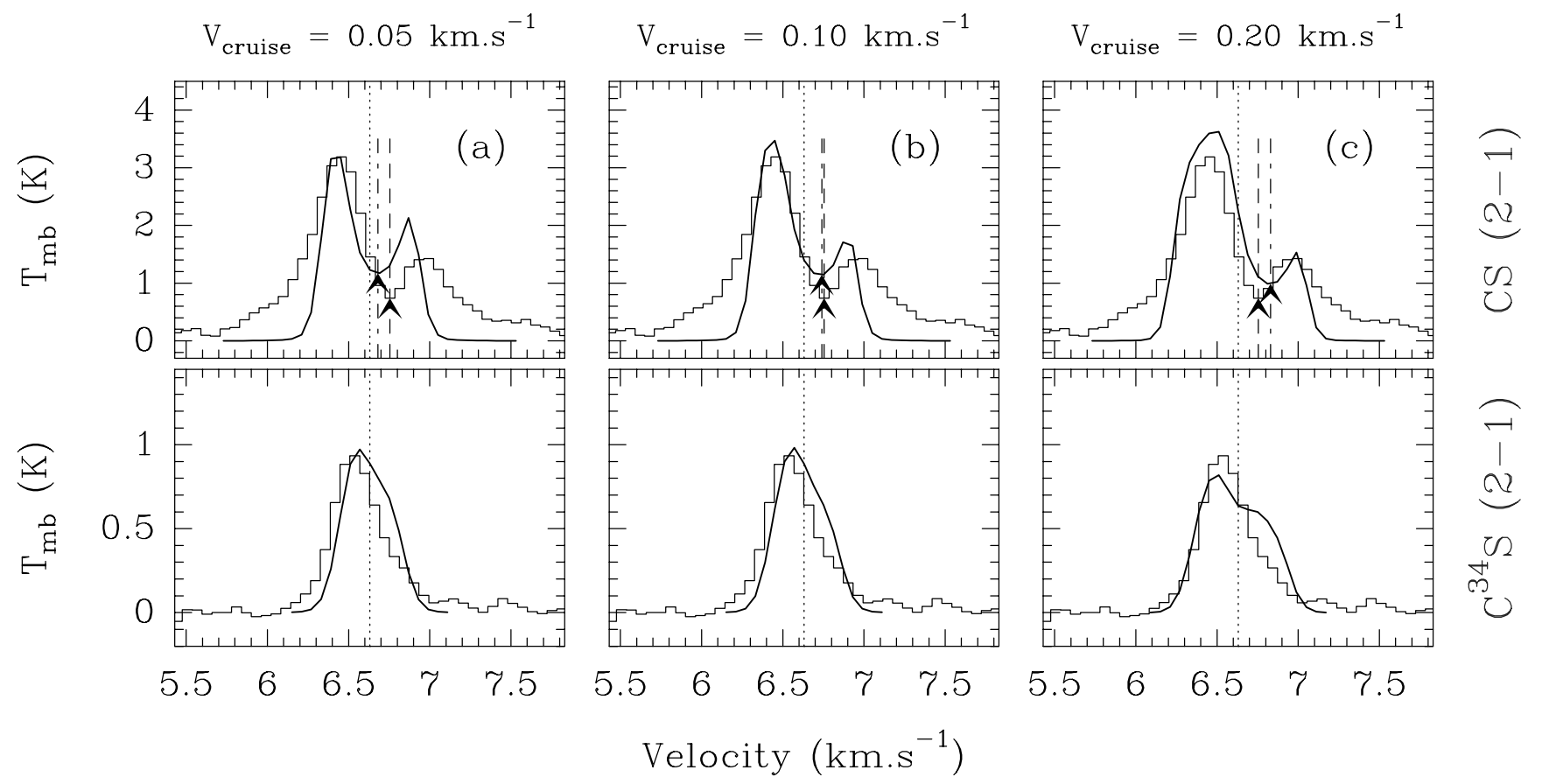

Fig. 9. Influence of the outer infall velocity field on the $\mathrm{CS}(2-1)$ (upper row) and $\mathrm{C}^{34} \mathrm{~S}(2-1)$ (lower row) line profiles. Synthetic spectra (solid curves) corresponding to a range of model infall velocities are superimposed on the spectra observed at the central position of IRAM 04191 (histograms). All models have $v_{\text {inf }}=v_{0}=0.2 \mathrm{~km} \mathrm{~s}^{-1}$ at $r_{0}=750 \mathrm{AU}, v_{\text {inf }} \propto r^{-0.5}$ for $r<r_{\text {break }}$, and $v_{\text {inf }}=v_{\text {cruise }}$ for $r>r_{\text {break. }}$. The models differ by the value of $r_{\text {break }}$, or equivalently by the value of the infall velocity at large radii, $v_{\text {cruise }} \equiv v_{0} \times\left(\frac{r_{\text {break }}}{r_{0}}\right)^{-0.5}: r_{\text {break }}=12000$ AU (i.e., $v_{\text {cruise }}=0.05 \mathrm{~km} \mathrm{~s}^{-1}$ ) in a), $r_{\text {break }}=3000 \mathrm{AU}$ (i.e., $v_{\text {cruise }}=0.1 \mathrm{~km} \mathrm{~s}^{-1}$ ) in b) (三 preferred model shown in Fig. 8), and $r_{\text {break }}=750 \mathrm{AU}$ (i.e., $v_{\text {cruise }}=0.2 \mathrm{~km} \mathrm{~s}^{-1}$ ) in c). The dotted vertical line marks the envelope systemic velocity as in Fig. 8, while the dashed and dash-dotted lines mark the velocities of the dips in the observed and synthetic CS(2-1) spectra, respectively. Note that the outer infall velocity $v_{\text {cruise }}$ is too small in model a) and too large in model c) to match the observed redshift of the $\mathrm{CS}(2-1)$ dip and the width of the $\mathrm{C}^{34} \mathrm{~S}(2-1)$ line.

$\sim 20$ toward the center as in Fig. 7c (see also Sect. 3.4). Such a depletion factor for CS is comparable to those observed in starless cores such as L1544 (e.g. Tafalla et al. 2002).

\subsection{Two regimes of infall}

In Fig. 8, we present a series of synthetic spectra emitted by a spherically symmetric model envelope with the input structure shown in Fig. 7 and our best estimate of the infall velocity field (shown by the solid line in Fig. 12a below). The model spectra are overlaid on the multitransition CS and $\mathrm{C}^{34} \mathrm{~S}$ spectra observed along the direction perpendicular to the outflow axis. The blue infall asymmetry of the model optically thick lines and the position of the $\mathrm{CS}(2-1)$ dip match the observations well. The widths of the optically thin lines are also well reproduced. The main shortcoming of the model is that it does not reproduce the fairly strong emission present in the wings of the observed CS spectra. As discussed in Sect. 3.5, we attribute these wings to the fraction of envelope material entrained by the outflow.

In order to determine the range of input model parameters that yield reasonably good fits to the observed CS and $\mathrm{C}^{34} \mathrm{~S}$ spectra, we have performed a comprehensive exploration of the parameter space, as illustrated in Figs. 9-11. Strong constraints on the infall velocity arise from the small optical depth lines, i.e., $C^{34} S(2-1)$ and $C^{34} S(3-2)$. The widths of these lines set firm upper limits to the absolute value of the infall velocity on the size scale of the beam. We obtain $v_{\text {inf }} \leq 0.15 \mathrm{~km} \mathrm{~s}^{-1}$ at $\sim 1750 \mathrm{AU}$ and $v_{\text {inf }} \leq 0.2 \mathrm{~km} \mathrm{~s}^{-1}$ at $\sim 1150 \mathrm{AU}$ from $\mathrm{C}^{34} \mathrm{~S}(2-$ 1) and $C^{34} S(3-2)$, respectively. On the other hand, the amplitude of the blue asymmetry seen in the self-absorbed CS(2-1) and CS(3-2) lines, as well as the redshifted position of the corresponding absorption dips (see Sect. 3.3), both require a relatively flat, extended infall velocity field with $v_{\text {inf }} \sim 0.10 \pm$ $0.05 \mathrm{~km} \mathrm{~s}^{-1}$ up to $r \sim 10000-12000 \mathrm{AU}$. The latter value approximately corresponds to the radius where the bulk of the absorption occurs in $\mathrm{CS}(2-1)$ and $\mathrm{CS}(3-2)$ (see Sect. 3.3). These constraints are illustrated in Fig. 9 which shows the effect of varying the infall velocity field on the central $\operatorname{CS}(2-1)$ and $C^{34} \mathrm{~S}(2-1)$ spectra. Three models are compared: the preferred model displayed in Fig. 8 is shown in the central panel (Fig. 9b), while models with lower and higher infall velocities in the outer part of the envelope are shown in the left a and right c panels, respectively. It can be seen that the position of the $\operatorname{CS}(2-1)$ absorption dip is not redshifted enough in model a and too redshifted in model $\mathrm{c}$ to match the observations. Furthermore, the $\mathrm{CS}(2-1)$ blue-to-red asymmetry is too weak in model a and the $\mathrm{C}^{34} \mathrm{~S}(2-1)$ line becomes too broad in model c compared to the observations. Only model b approximately reproduces the observed position of the $\mathrm{CS}(2-1)$ dip and the width of the $\mathrm{C}^{34} \mathrm{~S}(2-1)$ line.

In the context of a pure infall model, larger velocities $\left(v_{\text {inf }} \sim 0.2-0.4 \mathrm{~km} \mathrm{~s}^{-1}\right)$ in the inner $(r \sim 700 \mathrm{AU})$ part of the envelope are suggested by the broad linewidth of the central 

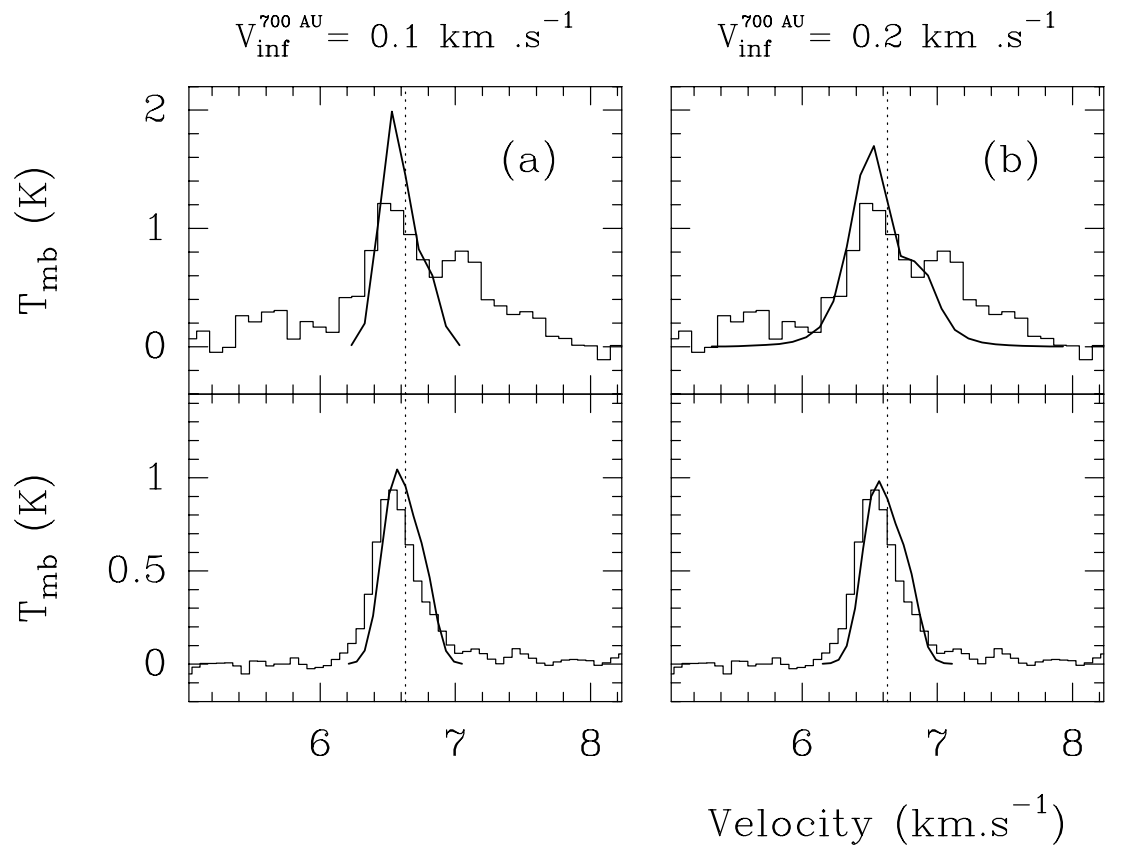

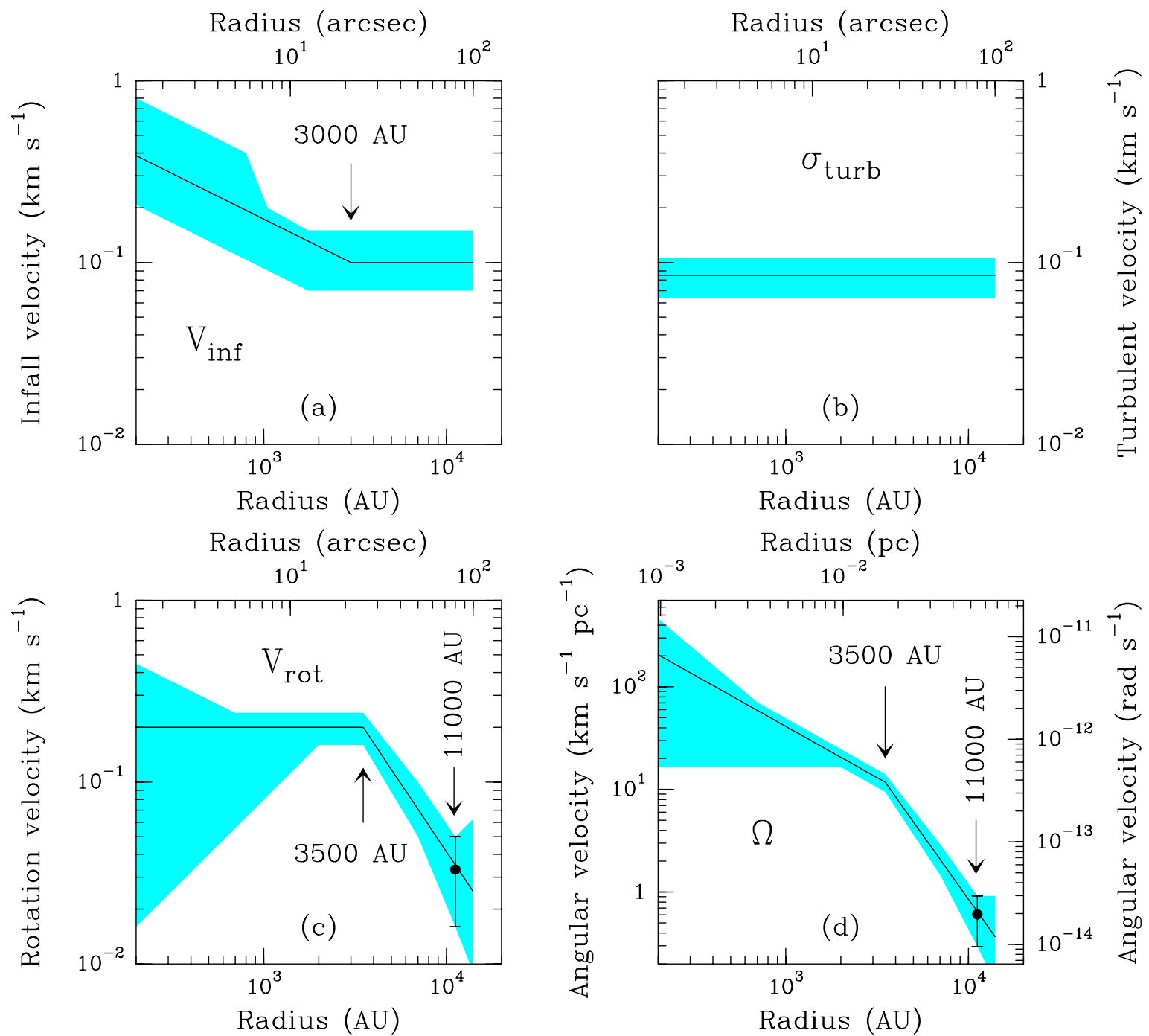

Fig. 12. Infall a), turbulence b), and rotation c) velocity fields inferred in the IRAM 04191 envelope based on our 1D (Sect. 4) and 2D (Sect. 5) radiative transfer modeling. The shaded areas show the estimated domains where the models match the CS and $\mathrm{C}^{34} \mathrm{~S}$ observations reasonably well. In a) and b), the solid lines show the infall velocity and turbulent velocity dispersion in both the 1D and 2D models (cf. Figs. 8 and 14, respectively) as a function of radius from envelope center. In c), the solid line represents the profile of the azimuthal rotation velocity in the $2 \mathrm{D}$ envelope model (cf. Fig. 14) as a function of radius from the outflow/rotation axis. The point with error bar at $11000 \mathrm{AU}$ corresponds to the velocity gradient observed in $\mathrm{C}^{18} \mathrm{O}$ (cf. Sect. 3.2). Panel d) shows the corresponding angular velocity profile.

by the width of the $\operatorname{CS}(2-1)$ and $\operatorname{CS}(3-2)$ dips is obtained for $\sigma_{\text {turb }}=0.085 \pm 0.02 \mathrm{~km} \mathrm{~s}^{-1}$ (cf. Fig. 11). This is equivalent to $\Delta v_{\text {turb }}^{F W H M}=\sigma_{\text {turb }} \times \sqrt{8 \ln 2}=0.20 \pm 0.05 \mathrm{~km} \mathrm{~s}^{-1}$ and corresponds to only half the thermal broadening of the mean molecular particle at $10 \mathrm{~K}$, showing that the IRAM 04191 envelope is "thermally-dominated" (see also Sect. 3.4) as are Taurus dense cores in general (e.g. Myers 1999).

The main conclusions of our 1D exploration of the parameter space are summarized in Figs. 12a and b, where the shaded areas represent the ranges of infall velocities a and turbulent velocity dispersion $b$ for which acceptable fits are found. Two infall regimes seem to stand out in Fig. 12a: the infall velocity is relatively large ( $v_{\text {inf }} \gtrsim 0.2 \mathrm{~km} \mathrm{~s}^{-1}$, supersonic) and increases toward the center for $r \lesssim 2000-3000 \mathrm{AU}$, while it is smaller and roughly uniform at $v_{\text {inf }} \sim 0.10 \pm 0.05 \mathrm{~km} \mathrm{~s}^{-1}$ between $\sim 2000-3000 \mathrm{AU}$ and $\sim 10000-12000$ AU. Given the density profile of Fig. 7a, such an infall velocity field implies a mass infall rate of $\dot{M}_{\text {inf }} \sim 3 \times 10^{-6} M_{\odot} \mathrm{yr}^{-1}$ at $r=1750 \mathrm{AU}$. (The density and velocity profiles shown in Figs. $7 \mathrm{a}$ and $12 \mathrm{a}$ are such that $\dot{M}_{\text {inf }}$ is roughly independent of radius.) Inside the $r \sim 11000 \mathrm{AU}$ region (where non-zero inward motions are inferred), the fraction of envelope mass with supersonic $\left(\gtrsim 0.16-0.2 \mathrm{~km} \mathrm{~s}^{-1}\right)$ infall motions is estimated to be only $\sim 1-10 \%$, depending on the exact value of the sound speed and exact form of the infall velocity profile (see Fig. 12a).

\section{Radiative transfer modeling: Simulations with infall and rotation}

\subsection{Quasi 2D simulations}

To account for the effects of rotation in the envelope (see Sect. 3.2 and Fig. 3), we have performed "quasi"-2D simulations with the following approximation. The non-LTE level 

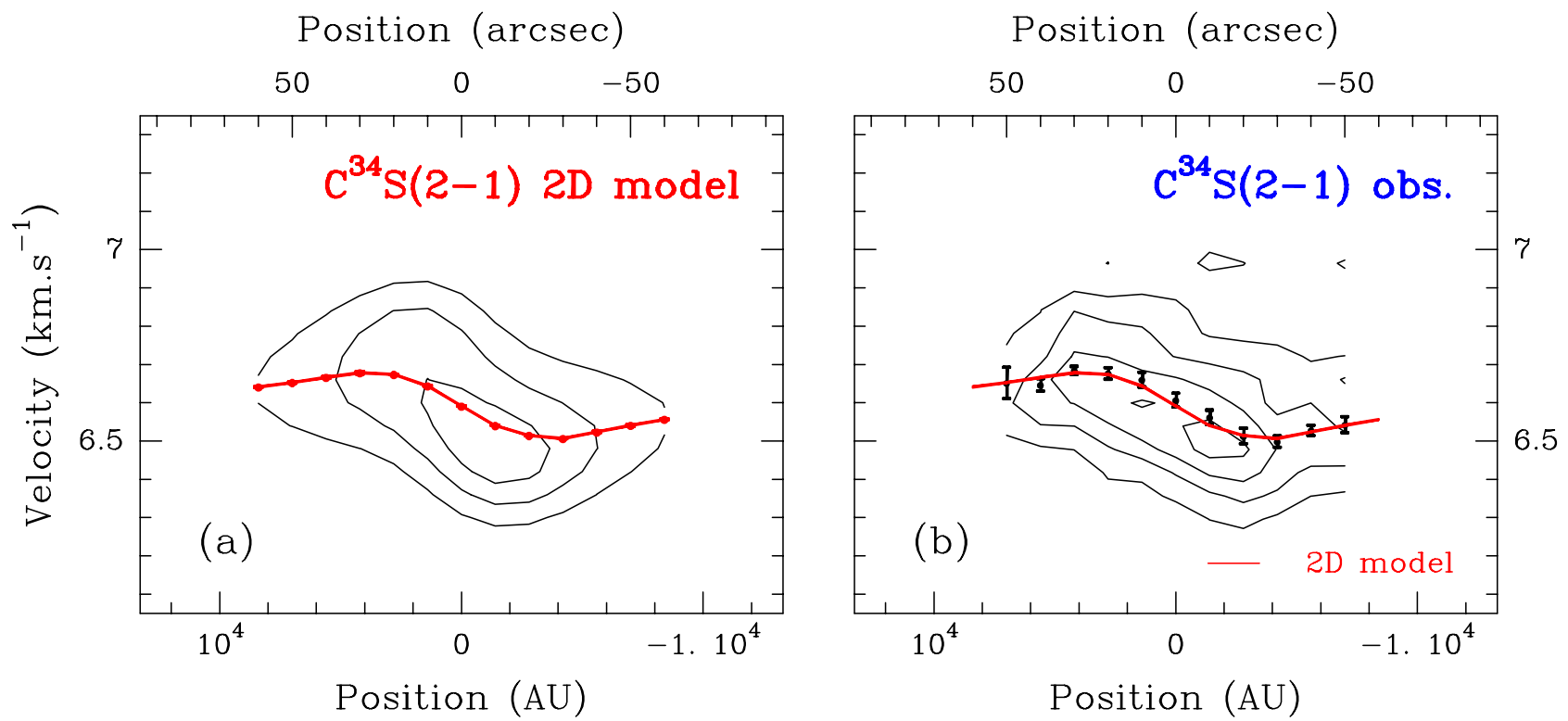

Fig. 13. a) $C^{34} S(2-1)$ position-velocity diagram of the "best-fit" $2 \mathrm{D}$ collapse model including differential rotation, taken along the direction perpendicular to the rotation axis. b) Observed $\mathrm{C}^{34} \mathrm{~S}(2-1)$ position-velocity diagram along the direction perpendicular to the outflow axis. In both diagrams, the dots represent the peak velocities derived from Gaussian fits at angular radii larger than $20^{\prime \prime}$ and from centroid (first moment) estimates for radii smaller than 20"; the curve shows the variation of the peak velocity (estimated in the same way) in the model.

populations are still calculated with a 1D Monte Carlo method (see Appendix) assuming a spherical envelope with the same characteristics as the model described in Sect. 4.3. We then add a cylindrical rotation velocity field to the $1 \mathrm{D}$ model of Sect. 4.3 and use the 2D version of the MAPYSO code to compute a proper radiative transfer integration along each line of sight. If we ignore departures from a spherical density distribution, this approach would remain strictly exact in the case of solidbody rotation, since the velocity difference between any couple of points projected on the axis joining these points is insensitive to the addition of a solid-body rotation component (cf. Ward-Thompson \& Buckley 2001). In practice, however, the rotation observed here departs from solid body and the density distribution is not spherical. We therefore assume that, to first order, the line excitation is much more sensitive to the density distribution (averaged over angles) than to the velocity field. (In particular, we have checked that the profiles of excitation temperature are essentially insensitive to the infall velocity field as long as the turbulent velocity dispersion is of the same order as the infall velocity.) The rotation velocity field has nevertheless important effects on the shape of the line profiles, which we properly take into account here.

\subsection{Two regimes of rotation}

The cylindrical rotation velocity field that we have added to the 1D spherical model of Sect. 4.3 is shown in Fig. 12c. The rotation axis is taken to coincide with the outflow axis (see Sect. 1.2 and Fig. 1).

Adding rotation yields two major improvements in the fits to the $\mathrm{CS}$ and $\mathrm{C}^{34} \mathrm{~S}$ spectra (see Figs. 13 and 14). First, the velocity gradient of the small optical depth $\mathrm{C}^{34} \mathrm{~S}(2-1)$ line along the direction perpendicular to the outflow is well reproduced, as shown in Fig. 13. Both the model $\mathrm{C}^{34} \mathrm{~S}(2-1)$ position-velocity diagram (Fig. 13a) and the centroid velocity curve agree well with the observations (Fig. 13b). Second, the blue asymmetry of the model CS(2-1) and CS(3-2) spectra is enhanced toward the north-west and attenuated toward the south-east, as seen on the observed spectra (see Sect. 3.3 and Fig. 14).

In agreement with the discussion of the position-velocity diagrams (Sect. 3.2 above), the present 2D modeling indicates that the envelope can be divided into two regions with distinct rotational characteristics (see the radial profiles derived for the rotational velocity and angular velocity in Figs. 12c and 12d, respectively). First, solid-body rotation is ruled out in the outer $3500<r<7000$ AU radius envelope, where a good fit to the centroid velocity curve is obtained with $v_{\text {rot }} \propto r^{-1.5 \pm 0.5}$, corresponding to an angular velocity $\Omega \propto r^{-2.5 \pm 0.5}$. Second, in the inner $r<3500$ AU radius region, our simulations suggest a velocity profile $v_{\text {rot }} \propto r^{0.1 \pm 0.4}$, i.e., $\Omega \propto r^{-0.9 \pm 0.4}$, although the form of the position-velocity diagram is significantly influenced by the finite resolution of the observations. A rotation velocity $v_{\text {rot }}=0.20 \pm 0.04 \mathrm{~km} \mathrm{~s}^{-1}$, corresponding to $\Omega=12 \pm 3 \mathrm{~km} \mathrm{~s}^{-1} \mathrm{pc}^{-1}$, is derived at a radius $r=3500 \mathrm{AU}$ after correcting for inclination ${ }^{6}$.

\section{Discussion: Comparison with collapse models}

In this section, we first summarize the predictions of collapse models and the main constraints derived from our observations (Sects. 6.1 and 6.2), and then discuss the applicability of various models to IRAM 04191 (Sects. 6.3 to 6.5). Some

\footnotetext{
6 The reason why the value of $12 \mathrm{~km} \mathrm{~s}^{-1} \mathrm{pc}^{-1}$ found here for $\Omega$ is larger than the apparent velocity gradient of $9 \mathrm{~km} \mathrm{~s}^{-1} \mathrm{pc}^{-1}$ given in Sect. 3.2 is that the 2D model shown in Fig. 13 properly accounts for the finite $\left(\sim 25^{\prime \prime}\right)$ resolution of the observations.
} 


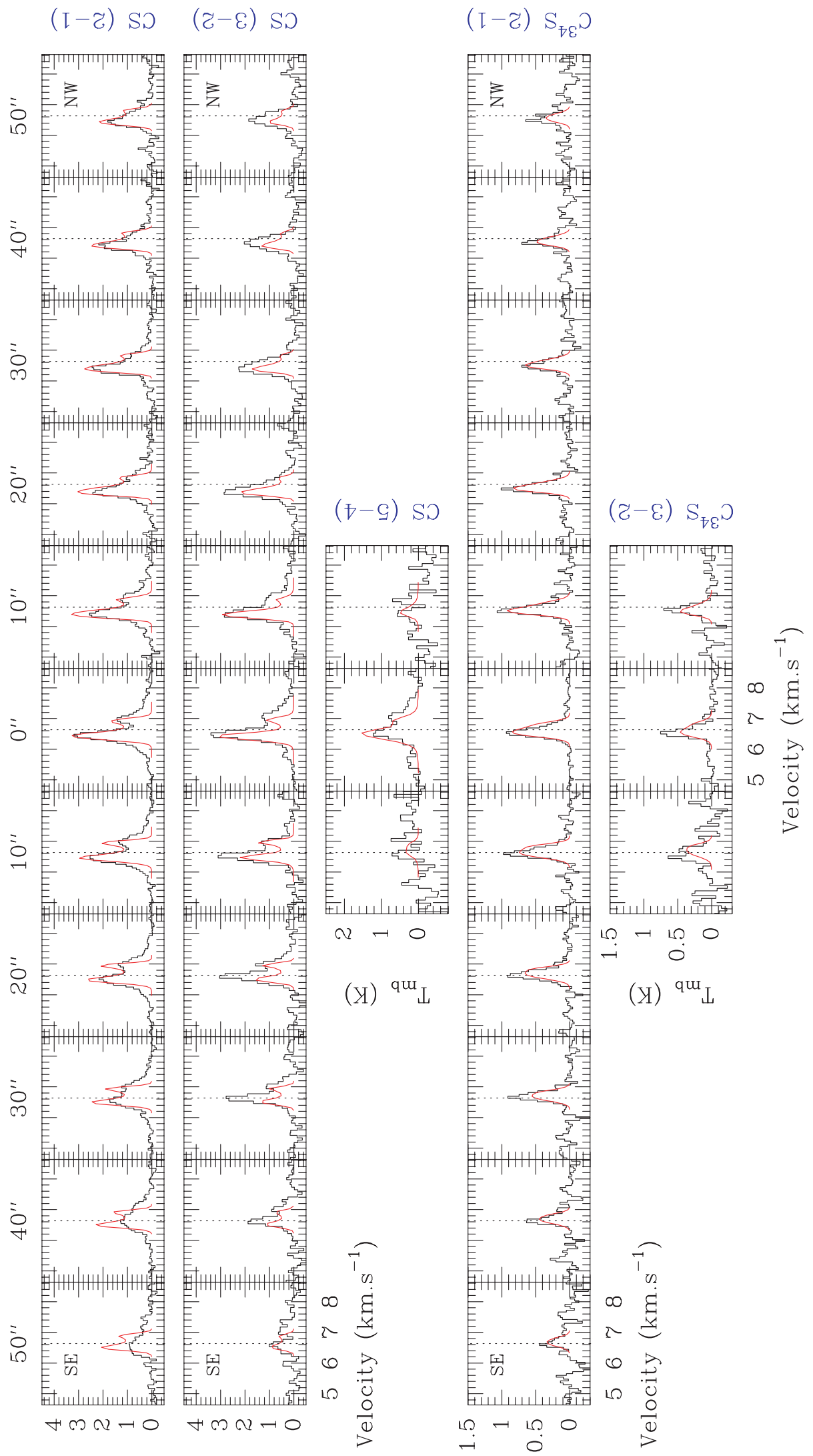

Fig. 14. Same as Fig. 8 but with synthetic spectra corresponding to the "best-fit" 2D model including differential rotation described in Sect. 5.2 (cf. Figs. 7 and 12 for model parameters). 
implications for the distribution and evolution of angular momentum during protostellar collapse are discussed in Sect. 6.6.

\subsection{Overview of model predictions}

Whitworth \& Summers (1985) have shown that there is a twodimensional continuum of similarity solutions to the problem of isothermal spherical collapse. In this continuum, the wellknown solutions proposed by Shu (1977) and Larson (1969)Penston (1969) represent two extreme limits. All isothermal similarity solutions share a universal evolutionary pattern. At early times $(t<0)$, a compression wave (initiated by, e.g., an external disturbance) propagates inward at the sound speed, $a_{\mathrm{s}}$, leaving behind it a $\rho(r) \propto r^{-2}$ density profile and a uniform infall velocity field. This compression wave has zero amplitude in the limiting case of the Shu "inside-out" collapse solution. At $t=0$, the compression wave reaches the center and a point mass forms which subsequently grows by accretion. At later times $(t>0)$, this wave is reflected into a rarefaction or expansion wave, propagating outward (also at the sound speed) through the infalling gas, and leaving behind it free-fall density and velocity distributions (i.e., $\rho(r) \propto r^{-1.5}$ and $v(r) \propto r^{-0.5}$ ). The various solutions can be distinguished by the absolute values of the density and velocity at $t \sim 0$. The Shu (1977) solution has the $\rho(r)=\left(a_{\mathrm{s}}^{2} / 2 \pi G\right) r^{-2}$ density distribution of a static $(v=0)$ singular isothermal sphere (SIS) at $t=0$, while the Larson-Penston (1969) solution is $\sim 4.4$ times denser and far from equilibrium $\left(v \approx-3.3 a_{\mathrm{s}}\right)$. The recent finding of inward motions of subsonic amplitude $\sim 0.02-0.10 \mathrm{~km} \mathrm{~s}^{-1}$, extended over $\sim 0.1 \mathrm{pc}(\sim 20000 \mathrm{AU})$ in the prestellar core L1544 (see Tafalla et al. 1998; Williams \& Myers 1999; Caselli et al. 2002a) suggests that true protostellar collapse in Taurus proceeds in a manner which is neither the Shu nor the LarsonPenston flow, and is perhaps more reminiscent of an intermediate similarity solution.

In practice, the initial conditions for fast protostellar collapse are not strictly self-similar and involve a density profile that is flat at small radii (e.g. Ward-Thompson et al. 1994; André et al. 1996) and bounded or sharp-edged at some outer radius $R_{\text {out }}$ (e.g. Motte et al. 1998; Bacmann et al. 2000) like a finite-sized Bonnor-Ebert isothermal sphere (e.g. Bonnor 1956; Alves et al. 2001). A number of recent numerical (magneto)hydrodynamic simulations or simplified analytical calculations attempt to describe the collapse in such a situation, either in the absence (e.g. Foster \& Chevalier 1993; Henriksen et al. 1997; Masunaga et al. 1998; Hennebelle et al. 2002) or in the presence (e.g. Tomisaka 1996; Basu 1997; Safier et al. 1997; Li 1998; Ciolek \& Königl 1998) of magnetic fields. The Larson-Penston similarity solution is found to describe the collapse quite satisfactorily near $t=0$ (at least for small radii), but the Shu solution is more adequate at intermediate $t \geq 0$ times, before the expansion wave reaches the edge of the initial, precollapse core.

When rotation is included, a rotationally-supported disk develops at the center of the infalling envelope during the accretion phase (i.e. at $t>0$ ). The size scale of this disk is determined by the centrifugal radius, $R_{\mathrm{C}}$, which defines the position where the centrifugal force balances gravity in the equatorial plane. Strong departures from a spherical density distribution and a purely radial inflow in the envelope are expected to occur on size scales of order (or smaller than) $R_{\mathrm{C}}$ (cf. Chevalier 1983; Hartmann 1998). Most collapse models predict that $R_{\mathrm{C}} \equiv j^{2} / \mathrm{Gm}$ should increase with time as material of higher and higher specific angular momentum $j=\Omega R^{2}$ falls in, but the exact dependence on time $t$, or alternatively accumulated central mass $m$, varies from model to model, according to the distributions of mass and angular momentum at $t=0$. For instance, $R_{\mathrm{C}}$ scales as $m^{3}$ or $t^{3}$ in the Shu model (TSC84), which assumes solid-body rotation at point mass formation. By contrast, the dependence of $R_{\mathrm{C}}$ on $m$ is only linear in the magnetically-controlled collapse model of Basu (1998) (see also Krasnopolsky \& Königl 2002).

\subsection{Summary of observational constraints}

The analysis of our line observations (Sects. 4 and 5) indicates that both the infall and rotation velocity fields of the IRAM 04191 envelope are characterized by an inner and an outer regime (see Fig. 12).

Our 1D radiative transfer simulations (Sect. 4) show that the infall velocity profile is flat with $v_{\text {inf }} \sim 0.1 \mathrm{~km} \mathrm{~s}^{-1}$ between $r_{\mathrm{i}} \sim 2000 \mathrm{AU}$ and $r_{\mathrm{i}, \mathrm{o}} \sim 10000-12000 \mathrm{AU}$. Higher infall velocities at radii $r<r_{\mathrm{i}}$ are suggested the CS observations, which are consistent with a free-fall velocity field $\left(v_{\text {inf }} \propto r^{-0.5}\right)$ at $r<r_{\text {i }}$. The width of the optically thin $\mathrm{C}^{34} \mathrm{~S}$ lines strongly constrains $v_{\text {inf }}$ to be $\lesssim 0.15 \mathrm{~km} \mathrm{~s}^{-1}$ at $r \sim r_{\mathrm{i}}$ (cf. Fig. 12a).

The position-velocity diagrams observed in optically thin lines (Sect. 3.2) show that the envelope is differentially rotating with an angular velocity profile $\Omega \propto r^{-2.5 \pm 0.5}$ between $r_{\mathrm{m}} \sim 3500 \mathrm{AU}$ and $r_{\mathrm{m}, \mathrm{o}} \sim 7000 \mathrm{AU}$, and $\Omega \sim 12 \mathrm{~km} \mathrm{~s}^{-1} \mathrm{pc}^{-1}$ at $r \sim r_{\mathrm{m}}$. Although the limited spatial resolution of our observations prevents us from deriving accurate values in the inner region, the rotation profile is definitely shallower for $r<r_{\mathrm{m}}$ (cf. Fig. 12d). A follow-up interferometric study is underway to provide more accurate constraints on the angular velocity in this inner region.

Although the two critical radii $r_{\mathrm{i}}$ and $r_{\mathrm{m}}$ differ by less than a factor of $\sim 2$, it is unclear whether they are physically related or not. The inner $r<r_{\mathrm{i}}$ envelope may correspond to the freefall region developing inside the expansion wavefront, while the outer $r>r_{\mathrm{i}}$ envelope may be dominated by the flat, extended inward velocity field set up by the compression wave at $t<0$ (see Sect. 6.1 above). If the current radius of the expansion wave is indeed $\sim r_{\mathrm{i}}$, then the age of the central protostellar object should be $t \sim r_{\mathrm{i}} / a_{\mathrm{s}} \lesssim 5 \times 10^{4} \mathrm{yr}$ (assuming a propagation speed $a_{\mathrm{s}} \sim 0.2 \mathrm{~km} \mathrm{~s}^{-1}$ ), in rough agreement with the estimated age of $\lesssim 3 \times 10^{4} \mathrm{yr}$ (Sect. 1.2). On the other hand, the fastly rotating $r<r_{\mathrm{m}}$ region may be a dynamically collapsing "supercritical" core in the process of decoupling from the ambient medium, and the outer $r_{\mathrm{m}}<r<r_{\mathrm{m}, \mathrm{o}}$ envelope may be a transition region between the protostar and the background cloud (see Sect. 6.5 below). 


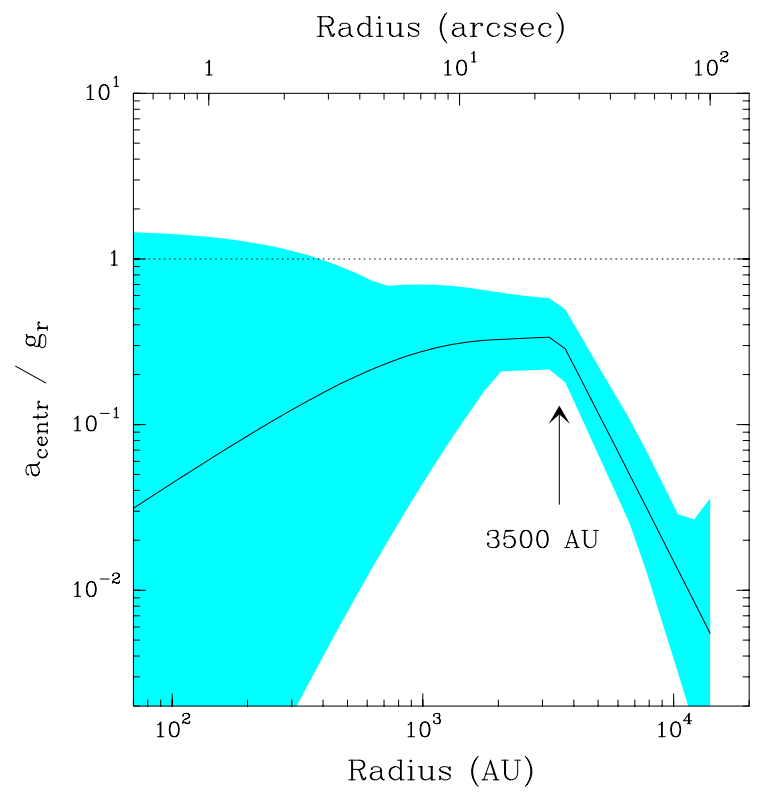

Fig. 15. Ratio of centrifugal to gravitational acceleration inferred in the IRAM 04191 envelope based on our 2D (Sect. 5) radiative transfer modeling. The dashed area shows the estimated domain where the models match the $\mathrm{CS}$ and $\mathrm{C}^{34} \mathrm{~S}$ observations reasonably well. The solid line shows the profile of $a_{\text {centr }} / g_{\mathrm{r}}$ in the 2D envelope model presented in Figs. 12-14, assuming $M_{\star}=0.05 M_{\odot}$.

\subsubsection{Centrifugal support}

Using our derived model for the structure and kinematics of the IRAM 04191 envelope (e.g. Figs. 7 and 12), we can estimate the dynamical importance of rotation in the envelope. The ratio of the centrifugal acceleration $a_{\text {centr }}=v_{\text {rot }}^{2} / r$ to the local gravitational field $g_{\mathrm{r}}=G \times\left[M_{\text {env }}(r)+M_{\star}\right] / r^{2}$ is shown in Fig. 15 . Here, $M_{\mathrm{env}}(r)$ is the envelope mass within radius $r$ corresponding to the density profile of Fig. $7 \mathrm{a}$, and $M_{\star}$ is the uncertain mass of the central protostellar object $\sim 0.03-0.1 M_{\odot}$ (cf. AMB99). We estimate a ratio $a_{\text {centr }} / g_{\mathrm{r}} \sim 0.4 \pm 0.2$ at the radius $r_{\mathrm{m}}=3500 \mathrm{AU}$ of the rapidly rotating inner envelope, showing that the centrifugal acceleration is a sizeable fraction of the gravitational acceleration in this region. Correspondingly, the ratio of rotational to gravitational energy is estimated to be fairly high, $\beta_{\text {rot }} \sim 0.1-0.2$, for the envelope material within $r \sim 3500-10000$ AU. Comparable values of $a_{\text {centr }} / g_{\mathrm{r}}$ and $\beta_{\text {rot }}$ are nevertheless obtained in some magnetic models of cloud collapse (see Fig. 4 of Basu \& Mouschovias 1994 and Sect. 6.5 below).

We estimate the centrifugal radius to be $R_{\mathrm{c}}<400 \mathrm{AU}$, assuming the maximum rotation velocity profile consistent with the observations (cf. Fig. 12c) and a stellar mass $M_{\star}=$ $0.03 M_{\odot}$. In principle, a large centrifugal radius should imply the presence of a large centrifugal disk around the central object. Stahler et al. (1994) have investigated the initial growth of protostellar disks in the context of the rotating collapse picture of TSC84 and have shown that a 3-component structure should develop inside $R_{\mathrm{c}}$ : a dense inner accretion disk of radius $R_{\text {disk }} \sim 0.34 R_{\mathrm{c}}$, a ring where material and angular momentum pile up at $R_{\text {disk }}$, and a low-density outer disk where material travels at high velocity between $R_{\mathrm{c}}$ and $R_{\text {disk }}$. If $R_{\mathrm{c}} \sim 200 \mathrm{AU}$, we may thus expect $R_{\text {disk }} \sim 70$ AU. Our $1.3 \mathrm{~mm}$ continuum interferometric observations set a firm upper limit to the radius of any dense inner circumstellar structure around IRAM 04191: $R_{\text {disk }}<10$ AU (cf. Sect. 3.1). This suggests that the actual centrifugal radius is significantly smaller than $200 \mathrm{AU}$ or else that the presence of a protostellar companion at $r \sim 30 \mathrm{AU}$ may have cleared a substantial gap in the inner accretion disk (cf. Jensen et al. 1996). Artymowicz \& Lubow (1994) show that, due to tidal disk truncation in binary systems, any individual disk must have an outer radius less than half the binary separation, and any circumbinary disk must have an inner radius more than roughly twice the binary separation. The non-detection of a dense $\sim 70 \mathrm{AU}$ radius disk by the PdBI interferometer could thus be accounted for by this effect if IRAM 04191 were a protobinary of separation $\sim 30 \mathrm{AU}$ (i.e. $\sim 0.2^{\prime \prime}$ ). The latter is plausible since Boss (1999) has shown that rotating, magnetic cloud cores typically fragment into binary protostars during collapse when $\beta_{\text {rot }}>0.01$ initially.

\subsection{Problems with the inside-out collapse model}

The inside-out, isothermal collapse model described by Shu (1977), and its 2D extension including rotation (TSC84), has been widely used to explain the infall spectral signature seen in the envelopes of low-mass protostars (e.g. Zhou et al. 1993; Myers et al. 1995; Choi et al. 1995; Zhou et al. 1996; Hogerheijde \& Sandell 2000). It accounts relatively well for the densities and accretion rates measured in Taurus protostellar envelopes (e.g. Ohashi 1999; Motte \& André 2001; this paper). This model is, however, inconsistent with the combined density and infall velocity profiles measured for IRAM 04191 (cf. Figs. 7a and 12a). An expansion wave radius $\gtrsim 10000 \mathrm{AU}$ is indeed required to reproduce the blue asymmetry of the central CS(2-1) spectrum in the context of the inside-out collapse picture. But such a large infall radius then implies high infall velocities at small radii $r \sim 1500 \mathrm{AU}$ which in turn yield central $C^{34} S(2-1)$ and $C^{34} S(3-2)$ spectra that are 2-3 times too wide compared to the observed linewidths. Conversely, a model with an expansion wave radius of only 2000 AU yields correct $C^{34} S(2-1)$ and $C^{34} S(3-2)$ linewidths but fails to reproduce the strong asymmetry observed in $\mathrm{CS}(2-1)$ and $\mathrm{CS}(3-2)$. It is clearly because of the absence of significant inward motions at $t<0$ that the inside-out collapse model cannot account for the infall velocity profile observed here. A similar inconsistency with the inside-out collapse model has been noted previously in prestellar cores such as L1544 by Tafalla et al. (1998) and Lee et al. (2001).

\subsection{Comparison with other thermal models of collapse}

With an age $\lesssim 3-5 \times 10^{4}$ yr for the central IRAM 04191 protostellar object (see Sects. 1.2 and 6.2) and a sound speed $a_{\mathrm{s}} \sim 0.19 \mathrm{~km} \mathrm{~s}^{-1}\left(T_{\mathrm{K}}=10 \mathrm{~K}\right)$, the radius of the expansion wave must be smaller than the inner radius $r_{\mathrm{i}} \sim 2000 \mathrm{AU}$ of the extended region where a flat infall velocity field is inferred (see Fig. 12a). The kinematics and density structure of the outer $r>r_{\mathrm{i}}$ envelope should thus still reflect the physical conditions at $t=0$. Accordingly, the observation of substantial infall velocities between $r_{\mathrm{i}}$ and $r_{\mathrm{i}, \mathrm{o}} \sim 11000 \mathrm{AU}$ points to collapse 
models that are more dynamical than the Shu solution and involve the propagation of a finite-amplitude compression wave prior to the formation of the central object (cf. Sect. 6.1 and Whitworth \& Summers 1985). On the other hand, the infall velocities derived in the outer $\left(r>r_{\mathrm{i}}\right)$ envelope are only subsonic (approximately half the sound speed), and clearly inconsistent with the supersonic infall velocities $\sim 3.3 a_{\mathrm{s}}$ characterizing the Larson-Penston isothermal similarity solution at $t=0$ (cf. Sect. 6.1). The infall velocity field derived here (cf. Fig. 12a) is suggestive of a moderately dynamical collapse model, intermediate between the Shu similarity solution (zero-amplitude compression wave at $t<0$ ) and the Larson-Penston solution (strong compression wave at $t<0$ ).

Qualitatively at least, such a moderately dynamical infall velocity field resembles that achieved during the collapse of a finite-sized, Bonnor-Ebert isothermal sphere. For instance, in their numerical simulations of the collapse of critically stable Bonnor-Ebert spheres (without magnetic field or rotation), Foster \& Chevalier (1993) found infall velocities at $t=0$ ranging from $3.3 a_{\mathrm{s}}$ near the origin to 0 at the outer boundary radius. Assuming that the IRAM 04191 dense core was initially a marginally stable Bonnor-Ebert sphere with a centerto-edge density contrast of $\sim 14$, the central density at the onset of collapse must have been $\sim 5 \times 10^{4} \mathrm{~cm}^{-3}$, given the typical outer density $\sim 3 \times 10^{3} \mathrm{~cm}^{-3}$ observed in prestellar cores (e.g. Bacmann et al. 2000). Adopting a temperature of $10 \mathrm{~K}$, Fig. 1 of Foster \& Chevalier (1993) then predicts an infall velocity at $t=0$ varying from $a_{\mathrm{s}} \sim 0.2 \mathrm{~km} \mathrm{~s}^{-1}$ at $\sim 6000$ AU to $0.5 a_{\mathrm{s}} \sim 0.1 \mathrm{~km} \mathrm{~s}^{-1}$ at $\sim 15000 \mathrm{AU}$. Even if this represents a somewhat stronger variation of infall velocity with radius than derived in the case of IRAM 04191 (Fig. 12a), the Foster \& Chevalier model clearly provides a much better fit to the observations than either the Shu or the Larson-Penston similarity solution. A definite problem with the critical Bonnor-Ebert model, however, is that as much as $44 \%$ of the envelope mass is predicted to flow in supersonically at $t=0$ (Foster \& Chevalier 1993), and progressively more mass at $t>0$. This is much larger than the $\sim 1-10 \%$ mass fraction derived in Sect. 4.3 for IRAM 04191. We conclude that spherically symmetric collapse models in which thermal pressure provides the only force opposing gravity tend to be too dynamical and are only marginally consistent with our observational constraints. It is likely that the inclusion of rotation in thermal models would improve the comparison with observations and may even account for the flattened shape of the protostellar envelope seen in Fig. 1 (P. Hennebelle, private communication). However, a generic feature of rotating thermal collapse models is that, due to simultaneous conservation of energy and angular momentum, they tend to predict similar forms for the rotation and infall velocity profiles at radii (much) larger than the centrifugal radius (e.g. Saigo \& Hanawa 1998). This is at variance with the steeply declining rotation velocity profile and flat infall velocity profile we observe beyond $3500 \mathrm{AU}$ (a radius much larger than the estimated centrifugal radius - see Sect. 6.2.1 above). In fact, the strong decline of the rotation velocity profile beyond $3500 \mathrm{AU}$ suggests that angular momentum is not conserved in the outer envelope. In the next subsection, we propose that this results from magnetic braking.

\subsection{Comparison with magnetically-controlled collapse models}

Magnetic ambipolar diffusion models (e.g. Ciolek \& Mouschovias 1994; Basu \& Mouschovias 1994, 1995a,b - hereafter BM94, BM95a, BM95b) are another class of models which yield non-zero inward velocities in an extended region prior to point mass formation. Ambipolar diffusion has been invoked by Ciolek \& Basu (2000) to explain the extended inward motions observed in the Taurus prestellar core L1544 (see Tafalla et al. 1998; Williams \& Myers 1999; Caselli et al. 2002a). The models start from a magnetically subcritical cloud, initially supported against gravitational collapse by a static magnetic field, and predict an evolution in two phases. During the first, quasistatic phase, the subcritical cloud contracts along directions perpendicular to the field lines through ambipolar diffusion. The gravitationally-induced inward drift of the neutral species is slowed down by collisions with the ions which are well coupled to the magnetic field. The central mass to magnetic flux ratio increases with time, until it reaches the critical value for collapse, $(1 / 2 \pi) G^{-0.5}$. The inner region of the cloud then becomes magnetically supercritical and collapses dynamically, while the outer envelope remains subcritical. This effect introduces a spatial scale in the collapse process, which corresponds to the boundary between the magnetically supercritical inner core and the subcritical outer envelope. These two regions are characterized by distinct rotational properties. The supercritical inner core evolves with conservation of angular momentum and rapidly spins up. It achieves a power-law angular velocity profile $\Omega(r) \propto \Sigma(r) \propto 1 / r$ at $t=0$, where $\Sigma$ is the (mass) column density (e.g. Basu 1997). By contrast, due to magnetic braking, the subcritical envelope loses (part of) its angular momentum on the timescale $\tau_{J} \approx t_{\mathrm{ff}}^{\mathrm{b}}$ (where $t_{\mathrm{ff}}^{\mathrm{b}}$ is the free-fall time at the density of the background - cf. Spitzer 1978 and Tomisaka 2000) and is progressively brought to near corotation with the background medium, assumed to rotate at the uniform rate $\Omega_{b}$ (e.g. BM94). This separation generates a break in the angular velocity profile at the radius $R_{\text {crit }}$ of the magnetically supercritical core.

Qualitatively at least, the rotation and infall profiles of the IRAM 04191 envelope (see Fig. 12 and Sect. 6.2) can be accounted for in the framework of such magnetic models ${ }^{7}$ if we identify $R_{\text {crit }}$ with the radius $r_{\mathrm{m}} \sim 3500$ AU beyond which the observed rotation profile exhibits a marked steepening (cf. Fig. 12c). Indeed, the angular velocity profile of the inner $r<r_{\mathrm{m}}$ region is consistent with the $\Omega \propto 1 / r$ power law expected in the supercritical core at $t=0$. Furthermore, the models predict a steepening of the rotation profile beyond $R_{\text {crit }}$ when magnetic braking does not have enough time to bring the system to corotation with the background before the formation

\footnotetext{
${ }^{7}$ Strictly speaking, the ambipolar diffusion models of Basu \& Mouschovias describe the evolution of core properties only during the prestellar phase $(t<0)$, while the properties of the IRAM 04191 envelope beyond $r_{\mathrm{i}} \sim 2000$ AU are representative of the physical conditions at $t \approx 0$ (cf. Sect. 6.4). However, the models are easily extrapolated to $t=0$ by considering the limiting profiles achieved for an infinite central density (cf. Basu 1997, 1998).
} 
of the supercritical core. In the parameter study presented by BM95a and BM95b, this happens in models 6 and 8, for two different reasons. First, if the cloud is already critical (or close to critical) initially near the center (but still subcritical in its outer parts) as in model 6 , there is no quasistatic phase and the dynamical contraction of the supercritical core starts right away on a timescale $\sim t_{\text {ff }}^{\mathrm{c}}$ (free-fall time at cloud center) shorter than $\tau_{J}$. This produces a transition region beyond $R_{\text {crit }}$ with a steep angular velocity profile between the supercritical inner core and the background outer region (see Fig. 6b of BM95b). At the same time, the collapse of the supercritical core is retarded by the magnetic forces so that supersonic infall velocities develop only very close to the center (at $r \lesssim 0.01 \times R_{\text {crit }}$ ). Second, if the ionization fraction is low enough that the magnetic braking timescale is only slightly shorter than the ambipolar diffusion timescale as in model 8 of BM95a, then the supercritical core can begin its dynamical evolution with a rotational angular velocity much larger than that of the background. This also results in the apparition of a transition region with a steep rotation profile between the supercritical core and the external background (cf. Fig. 5 of BM95a). In model 8 of BM95a, the outer radius of the transition region is $\sim 3 \times R_{\text {crit }}$, which is consistent with the extent of the $\Omega \propto r^{-2.5}$ zone in Fig. 12d. In this model, the infall velocity becomes supersonic for $r \lesssim 0.3 \times R_{\text {crit }}$ and exhibits a relatively flat profile beyond $R_{\text {crit }}$, also in agreement with the observational constraints of Fig. 12a. The ionization degree $x_{i}=n_{i} / n\left(\mathrm{H}_{2}\right)$ assumed in the model is low $\left(2 \times 10^{-9}\right.$ at a density of $10^{6} \mathrm{~cm}^{-3}$ ), but comparable to the value derived at the center of the IRAM 04191 envelope in Sect. 3.6.

Quantitatively, however, it is more difficult to obtain a good match of the observations of IRAM 04191 with published ambipolar diffusion models. These models rotate a factor of 23-10 more slowly than does the IRAM 04191 envelope $^{8}$ and have supercritical core radii that are a factor $\sim 3-30$ bigger than the observed break radius $r_{\mathrm{m}} \sim 3500$ AU (cf. Table 2 of BM95b). Physically, the radius of the magnetically supercritical core corresponds to the Jeans length for the density $n_{\text {crit }}$ (or equivalently surface density $\Sigma_{\text {crit }}$ ) at which "decoupling" occurs, i.e., the density (or surface density) at which gravity overcomes magnetic support. In the disk-like geometry of ambipolar diffusion core models, the critical Jeans radius is $R_{\text {crit }}=a_{\mathrm{s}}{ }^{2} /\left(2 G \Sigma_{\text {crit }}\right)$ and one has $n_{\text {crit }}=\frac{\pi G}{2 \mu \mathrm{m}_{\mathrm{H}}} \times\left(\Sigma_{\text {crit }} / a_{\mathrm{s}}\right)^{2}=$ $\frac{\pi}{8 G \mu \mathrm{m}_{\mathrm{H}}} \times\left(a_{\mathrm{s}} / R_{\text {crit }}\right)^{2}$ (see BM95a). For $T_{\text {core }}=7 \mathrm{~K}$ and $R_{\text {crit }}=$ $3500 \mathrm{AU}$, this gives $N_{\text {crit }} \equiv \frac{\Sigma_{\text {crit }}}{\mu \mathrm{m}_{\mathrm{H}}} \sim 9.3 \times 10^{21} \mathrm{~cm}^{-2}$ and $n_{\text {crit }} \approx 1.4 \times 10^{5} \mathrm{~cm}^{-3}$, the latter being remarkably similar to the volume density estimated at $r=3500 \mathrm{AU}$ in the envelope (as expected in the models - see, e.g., Fig. 6a of BM95b). If the mass-to-flux ratio $M / \Phi=\Sigma / B$ is just critical in the supercritical core, then the magnetic field strength of the core should be $B_{\text {crit }}=2 \pi G^{1 / 2} \Sigma_{\text {crit }} \sim 60 \mu \mathrm{G}$ at $r \sim 3500 \mathrm{AU}$. The reason why

\footnotetext{
${ }^{8}$ Although rotating more slowly than the IRAM 04191 envelope, some of the models are characterized by high ratios of centrifugal to gravitational acceleration at the supercritical radius, comparable to the $0.4 \pm 0.2$ ratio observed here (cf. model 1 of BM94 and model 6 of BM95b). Qualitatively, a faster initial rotation rate is not expected to change the evolution significantly compared to model 6 of BM95b (S. Basu, private communication).
}

published magnetic models have larger values of $R_{\text {crit }}$ is that their critical "decoupling" densities and field strengths are typically lower than these estimates by factors $\sim 10$ and $\sim 3$, respectively. Direct Zeeman measurements suggest that the magnetic field strength is only $\sim 10 \mu \mathrm{G}$ in the low-density $\left(\sim 10^{4} \mathrm{~cm}^{-3}\right)$ outer parts of prestellar cores such as L1544 (Crutcher 1999; Crutcher \& Troland 2000). Only relatively poor upper limits $(<100 \mu \mathrm{G})$ exist for the field strength in the central parts of these cores (Levin et al. 2001). Albeit quite large, the above value $B_{\text {crit }} \sim 60 \mu \mathrm{G}$, which refers to the $\geq 10^{5} \mathrm{~cm}^{-3}$ region, thus remains realistic. Furthermore, it should be noted that published ambipolar diffusion models include only a static magnetic field and do not take turbulent support into account. If a turbulent magnetic field is present, then a weaker static field may be sufficient to yield a decoupling density as high as $n_{\text {crit }} \sim 10^{5} \mathrm{~cm}^{-3}$. In this case, the observed radius of "decoupling" 3500 AU would correspond to the cutoff wavelength for MHD waves, i.e., $\lambda_{A} \sim 6200 \mathrm{AU} \times\left(\frac{B}{10 \mu \mathrm{G}}\right) \times\left(\frac{n_{\mathrm{H}_{2}}}{3 \times 10^{3} \mathrm{~cm}^{-3}}\right)^{-1}$ (cf. Mouschovias 1991), and the IRAM 04191 dense core would have initially formed through the dissipation of MHD turbulence (e.g. Nakano 1998; Myers 1998) rather than ambipolar diffusion.

\subsection{Evolution of angular momentum during protostar formation}

It is instructive to compare the rotational properties of the IRAM 04191 envelope with the characteristics of other prestellar and protostellar objects in Taurus. Our 2D radiative transfer modeling indicates a rotational velocity $v_{\text {rot }}=0.20 \pm$ $0.04 \mathrm{~km} \mathrm{~s}^{-1}$ at $r=3500 \mathrm{AU}$, using an inclination angle of $i=50^{\circ}$ (cf. Sect. 5.2). This corresponds to a local specific angular momentum $j=3.4 \times 10^{-3} \mathrm{~km} \mathrm{~s}^{-1} \mathrm{pc}$ at $r_{\mathrm{m}}=3500 \mathrm{AU}$ (roughly equal to the mean half-power radius of the $\mathrm{N}_{2} \mathrm{H}^{+}$ dense core: $F W H M \sim 8800 \mathrm{AU} \times 5200 \mathrm{AU})$. Quite remarkably, the mean specific angular momentum $J / M \sim 2 \times 10^{-3} \mathrm{~km} \mathrm{~s}^{-1} \mathrm{pc}$ we measure inside the rapidly rotating inner $r<r_{\mathrm{m}}$ envelope of IRAM 04191 is very similar to the nearly constant $\sim 10^{-3} \mathrm{~km} \mathrm{~s}^{-1} \mathrm{pc}$ value found by Ohashi (1999) for a number of small-scale "envelopes" and "disks" around Taurus Class I sources. More precisely, in the diagram of specific angular momentum versus radius presented by Ohashi et al. (1997b) (see their Fig. 6), IRAM 04191 lies close to the intersection between the "dense core" regime, where the angular velocity is approximately locked to a constant background value $\Omega_{b} \sim 1-2 \mathrm{~km} \mathrm{~s}^{-1} \mathrm{pc}^{-1}$ (presumably as a result of magnetic braking - see BM94 and Sect. 6.5 above), and the "protostellar" regime, where the specific angular momentum is roughly constant in time ${ }^{9}$ (cf. Fig. 16). According to Ohashi et al. (1997b), the transition between these two regimes at a radius $\sim 5000 \mathrm{AU}$

\footnotetext{
9 Assuming that all Taurus sources follow a similar time evolution, Fig. 16 may be viewed as an evolutionary diagram where the radius plotted on the $x$-axis represents the "contraction state" of a core as a function of time. This is conceptually different from a plot showing the spatial distribution of angular momentum/velocity in a given core at a given time (such as Fig. 1c of Basu 1997 or Fig. 12d of the present paper).
} 


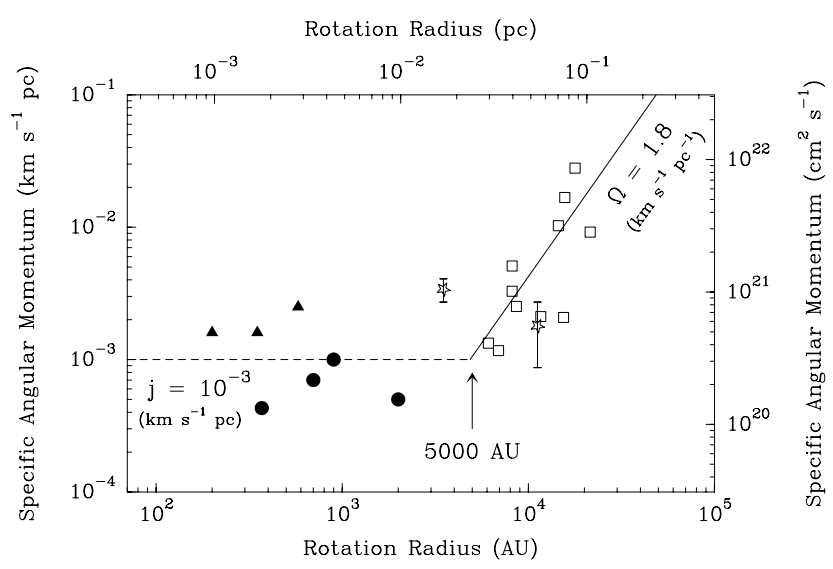

Fig. 16. Specific angular momentum as a function of radius for the Taurus $\mathrm{NH}_{3}$ dense cores of Goodman et al. (1993) (open squares) and the rotating "envelopes" and "disks" observed by Ohashi and collaborators around Taurus Class I sources (filled circles and filled triangles, respectively). The horizontal dashed line marks the constant specific angular momentum obtained by Ohashi (1999) for his sample of Class I sources; the solid line, corresponding to a constant angular velocity $\Omega=1.8 \mathrm{~km} \mathrm{~s}^{-1} \mathrm{pc}^{-1}$, shows the best-fit linear correlation determined by us for the Taurus $\mathrm{NH}_{3}$ cores of Goodman et al. (1993). The locations of the fastly rotating inner envelope $(r \sim 3500 \mathrm{AU})$ and more slowly rotating outer envelope $(r \sim 11000$ AU) in IRAM 04191 are marked by open stars, based on the present study. (Adapted from Ohashi 1999.)

(i.e., $\sim 0.03 \mathrm{pc}$ ) characterizes the size scale for dynamical collapse, inside which evolution proceeds with near conservation of angular momentum. Interestingly, this size scale is comparable to the radius $r_{\mathrm{m}} \sim 3500 \mathrm{AU}$ found here for the rapidly rotating inner envelope of IRAM 04191. Our suggestion that the inner envelope is a magnetically supercritical core decoupling from a subcritical environment (Sect. 6.5 above) is thus fully consistent with the finding and interpretation of Ohashi et al. (1997b).

\section{Summary and conclusions}

We have carried out a detailed study of the structure and kinematics of the envelope surrounding the Class 0 protostar IRAM 04191 in Taurus. Our main results and conclusions are as follows:

1. Extended, subsonic infall motions with $v_{\text {inf }} \sim 0.5 a_{\mathrm{s}} \sim$ $0.1 \mathrm{~km} \mathrm{~s}^{-1}$, responsible for a marked "blue infall asymmetry" in self-absorbed CS and $\mathrm{H}_{2} \mathrm{CO}$ lines, are present in the bulk of the envelope, up to at least $r_{\mathrm{i}, \mathrm{o}} \sim$ 10000-12000 AU. The observations are also consistent with larger infall velocities scaling as $v_{\text {inf }} \propto r^{-0.5}$ in an inner region of radius $r_{\mathrm{i}} \approx 2000 \mathrm{AU}$. The corresponding mass infall rate is estimated to be $\dot{M}_{\text {inf }} \sim 2-3 \times a_{\mathrm{s}}^{3} / G \sim$ $3 \times 10^{-6} M_{\odot} \mathrm{yr}^{-1}$.

2. The protostellar envelope is differentially rotating with an angular velocity profile $\Omega \propto r^{-2.5 \pm 0.5}$ between $r_{\mathrm{m}} \approx$ $3500 \mathrm{AU}$ and $r_{\mathrm{m}, \mathrm{o}} \sim 7000 \mathrm{AU}$. The rotation profile is shallower, albeit more poorly constrained, in the inner $r<r_{\mathrm{m}}$ region, i.e., $\Omega \propto r^{-0.9 \pm 0.4}$. The angular velocity is estimated to be $\Omega \sim 12 \mathrm{~km} \mathrm{~s}^{-1} \mathrm{pc}^{-1}$ at $r \sim 3500 \mathrm{AU}$ and only $\Omega \lesssim 0.5-1 \mathrm{~km} \mathrm{~s}^{-1} \mathrm{pc}^{-1}$ at $r \sim 11000$ AU. The present value of the centrifugal radius is estimated to be less than 400 AU.

3. The extended infall velocity profile is inconsistent with the inside-out collapse picture of Shu et al. (1987) and only marginally consistent with isothermal collapse models starting from marginally stable equilibrium Bonnor-Ebert spheres. The latter tend to produce somewhat faster infall velocities than are observed.

4. The contrast observed between the (steeply declining) rotation velocity profile and the (flat) infall velocity profile beyond $r_{\mathrm{m}} \approx 3500 \mathrm{AU}$ suggests that angular momentum is not conserved in the outer envelope. This is difficult to account for in the context of non-magnetic collapse models.

5. Based on a qualitative comparison with magnetic ambipolar diffusion models of cloud collapse (e.g. BM94), we propose that the rapidly rotating inner envelope of IRAM 04191 corresponds to a magnetically supercritical core decoupling from an environment still supported by magnetic fields and strongly affected by magnetic braking. In this view, the outer $\left(r_{\mathrm{m}}<r<r_{\mathrm{m}, \mathrm{o}}\right)$ envelope represents a transition region between the forming protostar and the slowly rotating ambient cloud. Although published ambipolar diffusion models have difficulty explaining supercritical cores as small as $R_{\text {crit }} \sim 3500 \mathrm{AU}$, we speculate that more elaborate versions of these models, including the effects of MHD turbulence in the outer envelope, would be more satisfactory.

6. Interestingly, the steepening of $\Omega(r)$ in IRAM 04191 occurs at a radius comparable to the $\sim 5000$ AU scale inside which the specific angular momentum of Taurus dense cores appears to be conserved (cf. Ohashi et al. 1997b and Fig. 16). Our results therefore support Ohashi et al. (1997b)'s proposal that $r \sim 5000$ AU represents the typical size scale for dynamical collapse in Taurus. More generally, we suggest that the rotation/infall properties observed here for IRAM 04191 are representative of the physical conditions prevailing in isolated protostellar envelopes shortly $\left(\sim 10^{4} \mathrm{yr}\right)$ after point mass formation.

Acknowledgements. We would like to thank Shantanu Basu for enlightening discussions on ambipolar diffusion models and Carl A. Gottlieb for providing his laboratory measurements of the CS and $\mathrm{C}^{34} \mathrm{~S}$ frequencies prior to publication. We acknowledge the contribution of Aurore Bacmann during the 1999 observing run at the $30 \mathrm{~m}$ telescope. We are grateful to the IRAM astronomers in Grenoble for their help with the Plateau de Bure interferometric observations. We would also like to thank Patrick Hennebelle and Pierre Lesaffre, as well as the referee, Phil Myers, for helpful comments.

\section{Appendix: Characteristics of the MAPYSO radiative transfer code}

The numerical code we have used first calculates the non-LTE level populations with a 1D (spherical) Monte-Carlo method (Bernes 1978, 1979). Radiative transfer along each line of sight and convolution with the antenna beam, approximated 
by a Gaussian, are then computed with the MAPYSO package (Blinder 1997). The latter works in both 1D and 2D geometry.

We have tested the Monte Carlo code for two test problems (1 and 2) available on the web page of the workshop on Radiative Transfer in Molecular Lines held in Leiden in May 1999 (http://www.strw.leidenuniv.nl/ $\sim$ radtrans/). These tests correspond to a low-abundance and high-abundance $\mathrm{HCO}^{+}$12-level problem, respectively, in the context of the Shu (1977) spherical collapse model. The level populations and the excitation temperatures computed by our Monte-Carlo code without any reference field (see Bernes 1979; Pagani 1998) agree quite well with those calculated by the workshop participants. The only significant difference occurs for the high-abundance case in the central region (inside $\sim 1300 \mathrm{AU}$ ) where our $\mathrm{HCO}^{+}(2-1),(3-2)$, and (4-3) excitation temperatures are lower by $\sim 20 \%$ compared to the main group results.

The CS and $\mathrm{C}^{34} \mathrm{~S}$ Monte-Carlo calculations reported in Sects. 4 and 5 used 9 levels and 27 concentric shells. This number of levels should be sufficient as the 9th level is $85 \mathrm{~K}$ above the ground level while the kinetic temperature in the envelope does not exceed $20 \mathrm{~K}$ (see Sect. 4.1). We used the CS collision rates computed in the $20-300 \mathrm{~K}$ range by Turner et al. (1992) and extrapolated these to 5-300 K with polynomials (Choi et al. 1995 and N. Evans, private communication). Each simulation was performed without any reference field and resulted from two successive Monte-Carlo runs. The first run started from LTE, used packets of 1000 model photons, and computed 100 iterations, reinitializing the counters after each iteration (see Bernes 1979). It converged rapidly but still suffered from a high level of statistical noise. The second run improved the convergence and reduced the noise level by starting from the output of the first run, computing 40 iterations with packets of 40000 model photons, and reinitializing the counters after each group of five iterations. We checked that this number of iterations was large enough to reach convergence on the populations of the first five levels with an accuracy better than a few percents.

\section{References}

Alves, J. F., Lada, C. J., \& Lada, E. A. 2001, Nature, 409, 159 André, P., Motte, F., \& Bacmann, A. 1999, ApJ, 513, L57 (AMB99) André, P., Ward-Thompson, D., \& Barsony, M. 1993, ApJ, 406, 122 André, P., Ward-Thompson, D., \& Barsony, M. 2000, in Protostars and Planets IV, ed. V. Mannings, A. P. Boss, \& S. S. Russell (Univ. of Arizona Press, Tucson), 59

André, P., Ward-Thompson, D., \& Motte, F. 1996, A\&A, 314, 625

Arquilla, R., \& Goldsmith, P. F. 1986, ApJ, 303, 356

Artymowicz, P., \& Lubow, S. H. 1994, ApJ, 421, 651

Bachiller, R., \& Pérez Guttiérrez, M. 1997, ApJ, 487, L93

Bacmann, A., André, P., Puget, J.-L., et al. 2000, A\&A, 361, 555

Basu, S. 1997, ApJ, 485, 240

Basu, S. 1998, ApJ, 509, 229

Basu, S., \& Mouschovias, T. C. 1994, ApJ, 432, 720 (BM94)

Basu, S., \& Mouschovias, T. C. 1995a, ApJ, 452, 386 (BM95a)

Basu, S., \& Mouschovias, T. C. 1995b, ApJ, 453, 271 (BM95b)

Beckwith, S. V. W., Sargent, A. I., Chini, R. S., \& Guesten, R. 1990, AJ, 99, 924
Benson, P. J., \& Myers, P. C. 1989, ApJSS, 71, 89

Bergin, E. A., \& Langer, W. D. 1997, ApJ, 486, 316

Bernes, C. 1978, Stockholm Observatory Report No. 15 erratum

Bernes, C. 1979, A\&A, 73, 67

Blinder, S. 1997, Thesis, Université de Bordeaux I

Bonnor, W. B. 1956, MNRAS, 116, 351

Boss, A. P. 1999, ApJ, 520, 744

Broguière, D., Neri, R., Sievers, A., \& Wiesemeyer, H. 2002, NIC Bolometer Users Guide, V 1.4-11

Buisson, G., Desbats, L., Duvert, G., et al. 2002, CLASS manual, version 4.0 (IRAM)

Burkert, A., \& Bodenheimer, P. 2000, ApJ, 543, 822

Cabrit, S., \& Bertout, C. 1990, ApJ, 348, 530

Caselli, P., Myers, P. C., \& Thaddeus, P. 1995, ApJ, 455, L77

Caselli, P., Walmsley, C. M., Terzevia, R., \& Herbst, E. 1998, ApJ, 499, 234

Caselli, P., Walmsley, C. M., Zucconi, A., et al. 2002a, ApJ, 565, 331

Caselli, P., Walmsley, C. M., Zucconi, A., et al. 2002b, ApJ, 565, 344

Ceccarelli, C., Hollenbach, D. J., \& Tielens, A. G. G. M. 1996, ApJ, 471,400

Chevalier, R. A. 1983, ApJ, 268, 753

Choi, M., Evans, N. J. II, Gregersen, E., \& Wang, Y. 1995, ApJ, 448, 742

Ciolek, G. E., \& Basu, S. 2000, ApJ, 529, 925

Ciolek, G. E., \& Königl, A. 1998, ApJ, 504, 257

Ciolek, G. E., \& Mouschovias, T. C. 1994, ApJ, 425, 142

Crutcher, R. M. 1999, ApJ, 520, 706

Crutcher, R. M., \& Troland, T. H. 2000, ApJ, 537, L139

Doty, S. D., \& Neufeld, D. A. 1997, ApJ, 489, 122

Evans, N. J. II 1999, ARA\&A, 37, 311

Evans, N. J. II, Rawlings, J. M. C., Shirley, Y. L., \& Mundy, L. G. 2001, ApJ, 557, 193

Foster, P. N., \& Chevalier, R. A. 1993, ApJ, 416, 303

Galli, D., \& Shu, F. H. 1993, ApJ, 417, 220

Goodman, A. A., Benson, P. J., Fuller, G. A., \& Myers, P. C. 1993, ApJ, 406, 528

Gottlieb, C. A., Myers, P. C., \& Thaddeus, P. 2002, in preparation

Guilloteau, S., Lucas, R., \& Bouyoucef, K. 2002, Mapping Cookbook IRAM Plateau de Bure Interferometer, version 1.3 (IRAM)

Hartmann, L. 1998, Accretion Processes in Star Formation, Cambridge Astrophysics Ser., vol. 32 (Cambridge University Press), 35

Hennebelle, P., Whitworth, A. P., Gladwin, P. P., \& André, P. 2002, MNRAS, in press

Henriksen, R. N., André, P., \& Bontemps, S. 1997, A\&A, 323, 549

Hogerheijde, M. R., \& Sandell, G. 2000, ApJ, 534, 880

Jensen, E. L. N., Mathieu, R. D., \& Fuller, G. A. 1996, ApJ, 458, 312

Jessop, N. E., \& Ward-Thompson, D. 2000, MNRAS, 311, 63

Kenyon, S. J., Calvet, N., \& Hartmann, L. W. 1993, ApJ, 414, 676

Klapper, G., Lewen, F., Gendriesch, R., Belov, S. P., \& Winnewisser, G. 2001, Z. Naturforsch., 56a, 329

Kramer, C., Stutzki, J., Röhrig, R., \& Corneliussen, U. 1998, A\&A, 329, 249

Krasnopolsky, R., \& Königl, A. 2002, ApJ, submitted [astro-ph/0205041]

Larson, R. B. 1969, MNRAS, 145, 271

Lee, C. W., Myers, P. C., \& Tafalla, M. 2001, ApJS, 136, 703

Levin, S. M., Langer, W. D., Velusamy, T., Kuiper, T. B. H., \& Crutcher, R. M. 2001, ApJ, 555, 850

Li, Z.-Y. 1998, ApJ, 493, 230

Li, Z.-Y., \& Shu, F. H. 1997, ApJ, 475, 237

Lucas, R. 1999, CLIC manual, version 4.1 (IRAM)

Masunaga, H., \& Inutsuka, S. I. 2000, ApJ, 531, 350 
Masunaga, H., Miyama, S. M., \& Inutsuka, S. 1998, ApJ, 495, 346

Motte, F., \& André, P. 2001, A\&A, 365, 440 (MA01)

Motte, F., André, P., \& Neri, R. 1998, A\&A, 336, 150

Mouschovias, T. C. 1991, ApJ, 373, 169

Myers, P. C. 1998, ApJ, 496, L109

Myers, P. C. 1999, in The Physics and Chemistry of the Interstellar Medium, ed. V. Ossenkopf, J. Stutzki, \& G. Winnewisser (GCA-Verlag Herdecke), 227

Myers, P. C., Bachiller, R., Caselli, P., et al. 1995, ApJ, 449, L65

Myers, P. C., Evans, N. J. II, \& Ohashi, N. 2000, in Protostars and planets IV, ed. V. Mannings, A. Boss, \& S. Russell (Tucson: Univ. Arizona Press), 217

Nakano, T. 1998, ApJ, 494, 587

Ohashi, N. 1999, in Star Formation 1999, ed. T. Nakamoto (Nobeyama Radio Observatory), 129

Ohashi, N., Hayashi, M., Ho, P. T. P., \& Momose, M. 1997a, ApJ, 475, 211

Ohashi, N., Hayashi, M., Ho, P. T. P., et al. 1997b, ApJ, 488, 317

Onishi, T., Mizuno, A., \& Fukui, Y. 1999, PASJ, 51, 257

Pagani, L. 1998, A\&A, 333, 269

Penston, M. V. 1969, MNRAS, 144, 425

Saigo, K., \& Hanawa, T. 1998, ApJ, 493, 342

Safier, P. N., McKee, C. F., \& Stahler, S. W. 1997, ApJ, 485, 660

Shu, F. 1977, ApJ, 214, 488

Shu, F. H., Adams, F. C., \& Lizano, S. 1987, ARA\&A, 25, 23

Spitzer, L., Jr. 1978, Physical Processes in the Interstellar Medium (New York: Wiley), 291

Stahler, S. W., Korycansky, D. G., Brothers, M. J., \& Touma, J. 1994, ApJ, 431341

Stutzki, J. , \& Güsten, R. 1990, 356, 513

Tafalla, M., Mardones, D., Myers, P. C., et al. 1998, ApJ, 504, 900
Tafalla, M., Myers, P. C., Caselli, P., Walmsley, C. M., \& Comito, C. 2002, ApJ, 569, 815

Tamura, M., Gatley, I., Waller, W., \& Werner, M. W. 1991, ApJ, 374, L25

Terebey, S., Shu, F. H., \& Cassen, P. 1984, ApJ, 286, 529 (TSC84)

Tomisaka, K. 1996, PASJ, 48, L97

Tomisaka, K. 2000, ApJ, 528, L41

Turner, B. E., Chan, K. W., Green, S., \& Lubowich, D. A. 1992, ApJ, 399,114

Ungerechts H., et al. 2000, in Imaging at Radio through Submillimeter Wavelengths, ed. J. G. Mangum, \& S. J. E. Radford (Astronomical Society of the Pacific), ASP Conf. Proc., 217, 190

Vrtilek, J. M., Gottlieb, C. A., \& Thaddeus, P. 1987, ApJ, 314, 716

Walker, C. K., Narayanan G., \& Boss, A. P. 1994, ApJ, 431, 767

Ward-Thompson, D., \& Buckley, H. D. 2001, MNRAS, 327, 955

Ward-Thompson, D., Motte, F., \& André, P. 1999, MNRAS, 305, 143

Ward-Thompson, D., Scott, P. F., Hills, R. E., \& André, P. 1994, MNRAS, 268, 276

Whitworth, A., \& Summers, D. 1985, MNRAS, 214, 1

Wild, W. 1999, The $30 \mathrm{~m}$ Manual, Pico Veleta, Spain, version 2.0 (IRAM)

Williams, J. P., \& Myers, P. C. 1999, ApJ, 518, L37

Wilner, D. J., Myers, P. C., Mardones, D., \& Tafalla, M. 2000, ApJ, 544. L73

Wilson, T. L., \& Rood, R. T. 1994, ARA\&A, 32, 191

Wootten, A., Wiseman, J., \& Fuller, G. 2001, AAS Meet. 199, \#60.02

Zhou, S. 1995, ApJ, 442, 685

Zhou, S., Evans, N. J. II, Kömpe, C., \& Walmsley, C. M. 1993, ApJ, 404, 232

Zhou, S., Evans, N. J. II, \& Wang, Y. 1996, ApJ, 466, 296

Zucconi, A., Walmsley, C. M., \& Galli, D. 2001, A\&A, 376, 650 